\title{
Formulation of generalised mass transfer correlations for blood oxygenator design
}

\author{
Kenny W. Q. Low * \\ Email: k.w.q.low@swansea.ac.uk \\ Raoul van Loon \\ Email: r.vanloon@swansea.ac.uk \\ Samuel A. Rolland \\ Email: s.rolland@swansea.ac.uk \\ Johann Sienz \\ Email: j.sienz@swansea.ac.uk
}

\begin{abstract}
Advanced Sustainable Manufacturing Technologies (ASTUTE 2020) Operation, College of Engineering, Swansea University, Bay Campus, Fabian Way, Swansea SA1 8EN, United Kingdom
\end{abstract}

\begin{abstract}
This paper numerically investigates non-Newtonian blood flow with oxygen and carbon dioxide transport across and along an array of uniformly square and staggered arranged fibres at various porosity $(\varepsilon)$ levels, focussing on a low Reynolds number regime $($ Re $<10)$. The objective is to establish suitable mass transfer correlations, expressed in the form of Sherwood number (Sh $=f(\varepsilon, R e, S c)$ ), that identifies the link from local mass transfer investigations to full-device analyses. The development of a concentration field is initially investigated and expressions are established covering the range from a typical deoxygenated condition up to a full oxygenated condition. An important step is identified where a cut-off point in those expressions is required to avoid any under-or over-estimation on the Sherwood number. Geometrical features of a typical commercial blood oxygenator is adopted and results in general show that a balance in pressure drop, shear stress and mass transfer is required to avoid potential blood trauma or clotting formation. Different definitions of mass transfer correlations are found for oxygen/carbon dioxide, parallel/transverse flow and square/staggered configurations, respectively. From this set of correlations, it is found that transverse flow has better gas transfer than parallel flow which is consistent with reported literature.
\end{abstract}


The mass transfer dependency on fibre configuration is observed to be pronounced at low porosity. This approach provides an initial platform when one is looking to improve the mass transfer performance in a blood oxygenator without the need to conduct any numerical simulations or experiments.

\section{Introduction}

An artificial lung, known as a blood oxygenator, has been developed to provide temporary respiratory support during surgery or healing process and potentially to replace the entire pulmonary system. To date, microporous hollow fibre membrane oxygenators have become the most common type of oxygenators in the market due to their large gas exchange surface area $[1,2]$. Blood enters the device through the inlet port and flows across or along a microporous hollow fibre bundle, where pure oxygen $\left(\mathrm{O}_{2}\right)$ or a mixture of room air and $\mathrm{O}_{2}$ flows through the lumen of the fibres and gas exchange occurs at the surface of the microporous membrane, before exiting the device through the outlet port. The current consideration aspects of designing blood oxygenators that motivate ongoing researches include minimising the blood contacting surface area of the fibres (reducing blood trauma) and priming volume whilst maximising the gas transfer per unit volume to achieve better oxygenation efficiency.

The overall mass transfer coefficient is one of the important elements to quantify mass transfer rate in an oxygenator for design evaluation and manufacture. The coefficient has three principal additive components; the first representing the gas-side, the second being the membrane-side and the last correspond to the blood-side [3]. The overall mass transfer coefficient is usually described through the mass transfer correlation which consists of non-dimensional mass transfer expressions, namely the Sherwood $(S h)$, Reynolds $(R e)$ and Schmidt $(S c)$ numbers. The expression is often seen in the form $S h=a R e^{b} S c^{0.33}$ for flow across bundles of hollow fibre membranes (transverse flow). The mass transfer correlation for flow along fibre bundles (parallel flow) appears in two different forms. The first form is similar to the expression for transverse flow where the flow and concentration profiles are considered to be fully developed [4]. However, when considering the development of flow and concentration profiles, the mass transfer correlation is commonly expressed as $S h=a\left(\operatorname{Re} \cdot d_{h} / L\right)^{b} S c^{0.33}$ or $S h=a G z^{b}$, using the Graetz number $\left(G z=\left(d_{h} / L\right) R e \cdot S c\right)$ [5]. The $a$ and $b$ parameters are commonly determined through fittings of experimental data conducted at a wide range of $R e$. These experiments are conducted with water using commercial oxygenators [6-13] or oxygenator prototypes [6,14-17]. To the authors' best knowledge, the development of mass transfer correlation for $\mathrm{O}_{2}$ is well established for transverse flow in blood oxygenators and no information is available in the development for $\mathrm{CO}_{2}$ mass transfer correlation. In contrast, limited experimental investigations have been conducted in the development of parallel flow mass transfer correlation of $\mathrm{O}_{2}$ for blood oxygenators. Summary of mass transfer correlations from the literature are tabulated in Tables 1 and 2 for transverse and parallel flows, respectively.

Although these experimental studies have led to practical correlations in the evaluation of blood oxygenators, it is well known that the parameters are dependent on the fibre arrangement, device porosity as well as the flow direction and as a result, they are unique to a particular device. Furthermore, detailed insight and understanding on the underlying phenomena is not yet attained. As a result, Computational Fluid Dynamics (CFD) is an attractive tool to compliment the experimental approaches. There are some researchers who investigate the mass transfer performance in blood oxygenators by employing a 
porous model approach [18-20]. However, the mass transfer correlations used in those works are adopted from the mentioned above which may not represent their respective device characteristics appropriately. Therefore, several research groups have focussed on a more fundamental flow and mass transfer analysis which involves investigation of blood flow and mass transfer past fibre bundles with different fibre configurations. Baker et al. [21] predicted gas transfer for non-Newtonian blood flow along a staggered arranged fibre array but no further analysis on local behaviour of flow and gas transport are performed. Dierickx et al. [22] studied the non-Newtonian blood flow behaviour (Casson model) past three different fibre configurations with porosity $(\varepsilon)$ and $R e$ ranging from 0.4 to 0.6 and 1 to 60 , respectively. They then extended their work [23] to include $\mathrm{O}_{2}$ transport in water and validated it against three commercial blood oxygenators. Taskin et al. [24] incorporated hollow-fibre membrane walls to study non-Newtonian blood flow behaviour and $\mathrm{O}_{2}$ transport past a single fibre, a staggered array of fibres and a mini-oxygenator prototype. Zierenberg et al. $[25,26]$ studied the fluid flow and mass transport past a circular cylinder for Newtonian and non-Newtonian fluid as a basis of designing an artificial lung device. Steady state and pulsatile flow were simulated and the effect between Newtonian and Casson (non-Newtonian) fluids on the flow behaviour and local $\mathrm{O}_{2}$ transport was investigated. The single fibre investigation is then extended to an array of square and staggered arranged fibres by Chan et al. [27] to study $\mathrm{O}_{2}$ and $\mathrm{CO}_{2}$ transport using a Casson fluid. The $\varepsilon$ and $R e$ range considered are $0.5,0.65$ and 0.8 and 1, 5 and 10 respectively. In both works, they showed that either pulsatility, increased $R e$ or decreased $\varepsilon$ improves gas transfer. Furthermore, they found that a staggered configuration achieves greater gas transfer and lower pressure drop than a square arrangement. Zierenberg et al. [28] subsequently performed investigations of $\mathrm{O}_{2}$ and $\mathrm{CO}_{2}$ transport for rectangular and staggered fibre configurations. The $\varepsilon$ range studied includes $0.5,0.61$ and 0.74 with $R e$ ranging between 1 and 20 . They reported the $S h$ for both steady and pulsatile flows are approximately equivalent. They also showed that pressure drop, shear stress and $S h$ decreases as $\varepsilon$ increases. More importantly, they deduced that a compromise between pressure drop, shear stresses and gas transport is required as high pressure drop and shear stresses lead to high $S h$ and vice versa.

Despite good efforts in analysing the oxygenation behaviour in fibre bundles, suitable mass transfer correlations have not been identified that can help bridge the gap from local mass transfer studies to full-device analysis. Therefore, in this current work, we seek to address this shortcoming. In this present context, we look to numerically investigate the non-Newtonian blood flow and gas transport behaviours on an array of fibres arranged in square and staggered manners at various porosity levels subject to transverse and parallel flows. This investigation focus on low $R e$ regime $(R e<10)$ which complies to the range reported by Zierenberg et al. [26] for commercial oxygenators. Furthermore, we aim to provide a generalised mass transfer correlation that can be utilised at an oxygenator scale to predict the oxygenation performance.

The layout of the current work is as follows: First, an outline of the methods is provided followed by the governing equations for fluid flow and oxygenation in fibre bundles. Numerical setup for the flow and oxygenation simulations are then described. All these are laid out in Section 2. The findings are presented in Section 3 which includes numerical validation, flow and mass transfer behaviour and finally, the identification and discussion on the developed mass transfer correlations. 


\begin{tabular}{lllllll}
\hline \hline Oxygenator & Gas & $\begin{array}{l}\text { Fibre dia. } \\
(\mu \mathrm{m})\end{array}$ & Porosity & Mass transfer correlation & Remarks & Literature \\
\hline Prototype & $\mathrm{O}_{2}$ & 400 & 0.93 & $S h=0.90 R e^{0.40} S c^{0.33}$ & - & {$[29]$} \\
Prototype & $\mathrm{O}_{2}$ & 400 & 0.30 & $S h=1.38 R e^{0.34} S c^{0.33}$ & - & {$[29]$} \\
Sarns 16310 & $\mathrm{O}_{2}$ & 300 & - & $S h=0.15 R e^{0.80} S c^{0.33}$ & $R e>2.5$ & {$[6]$} \\
Sarns 16310 & $\mathrm{O}_{2}$ & 300 & - & $S h=0.12 R e^{1.00} S c^{0.33}$ & $R e<2.5$ & {$[6]$} \\
Sarns Membrane & $\mathrm{O}_{2}$ & 290 & 0.58 & $S h=0.136 R e^{0.832} S c^{0.33}$ & - & {$[11]$} \\
Sarns Turbo & $\mathrm{O}_{2}$ & 380 & 0.45 & $S h=0.159 R e^{0.751} S c^{0.33}$ & - & {$[11]$} \\
Bentley Univox & $\mathrm{O}_{2}$ & 365 & 0.31 & $S h=0.256 R e^{0.779} S c^{0.33}$ & - & {$[11]$} \\
Prototype & $\mathrm{O}_{2}$ & 380 & 0.53 & $S h=0.52 R e^{0.29} S c^{0.33}$ & - & {$[30]$} \\
Sarns Turbo & $\mathrm{O}_{2}$ & 380 & 0.61 & $S h=0.47 \varepsilon \xi^{0.5} R e^{0.64} S c^{0.33}$ & $R e>6.5$ & {$[13]$} \\
Sarns Turbo & $\mathrm{O}_{2}$ & 380 & 0.61 & $S h=0.26 \varepsilon \xi^{0.5} R e^{1.00} S c^{0.33}$ & $R e<6.5$ & {$[13]$} \\
Prototype & $\mathrm{O}_{2}$ & 380 & 0.47 & $S h=0.46 R e^{0.76} S c^{0.33}$ & - & {$[12]$} \\
Prototype & $\mathrm{O}_{2}$ & 250 & 0.58 & $S h=0.46 R e^{0.76} S c^{0.33}$ & - & {$[12]$} \\
& & & & & & \\
\hline \hline
\end{tabular}

Table 1. Developed transverse flow mass transfer correlation for $\mathrm{O}_{2}$ from published literature. No information on the development of $\mathrm{CO}_{2}$ mass transfer correlations is identified. The terms $\varepsilon$ and $\xi$ denote the porosity and characteristic length respectively.

\begin{tabular}{lcccccc}
\hline \hline Oxygenator & Gas & $\begin{array}{l}\text { Fibre dia. } \\
(\mu \mathrm{m})\end{array}$ & Porosity & Mass transfer correlation & Remarks & Literature \\
\hline & & & & & \\
Prototype & $\mathrm{O}_{2}$ & 400 & 0.97 & $S h=1.25\left(R e \cdot d_{h} / L\right)^{0.93} \mathrm{Sc}^{0.33}$ & - & {$[29]$} \\
Prototype & $\mathrm{O}_{2}$ & 300 & 0.30 & $S h=0.019 \mathrm{Gz} z^{1.00}$ & $G z<60$ & {$[6]$} \\
& & & & & \\
\hline \hline
\end{tabular}

Table 2. Developed parallel flow mass transfer correlation for $\mathrm{O}_{2}$ from published literature. No information on the development of $\mathrm{CO}_{2}$ mass transfer correlations is identified. The terms $d_{h}$ and $L$ denote the hydraulic diameter and length of fibre respectively.

\section{Materials and Methods}

In this present work, the fibres are $380 \mu \mathrm{m}$ in diameter $(d)$ and assumed to be uniformly arranged periodically in which a representative unit cell can be utilised to provide a basic building block for the investigation of blood flow and mass transfer. Fibres in the unit cell are considered to be arranged in two different idealised manners, namely square and staggered (equilateral triangle), illustrated in Figure 1. The porosity of the unit cell adopted ranges from 0.3 to 0.7 in increments of 0.1 which covers the typical range for commercial oxygenators [26]. Furthermore, the Reynolds number range in this investigation is assumed to be in low regime $(\operatorname{Re} \approx 0.1-10)$ which is also within the typical operating conditions found to be ranging from 0.6 to 12 [26]. 


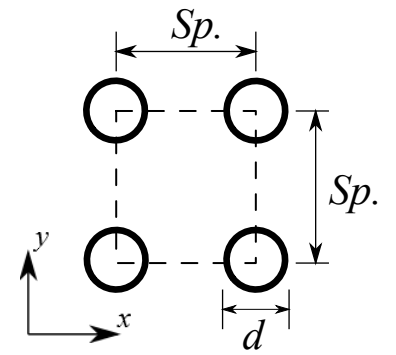

(a) Square

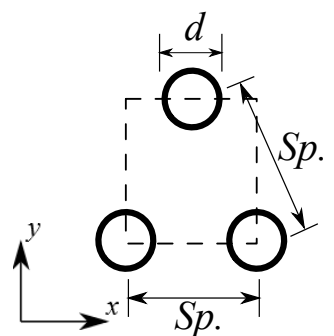

(b) Staggered

Fig. 1. Fibre bundles in the oxygenators which can be represented by a unit cell of ordered (a) square and (b) staggered arrays of cylinders. Illustration adopted from [31]. The annotations $d$ and $S p$. are the fibre diameter and spacing respectively.

\subsection{Governing equations for fluid flow}

The conservation of mass and momentum for an incompressible steady flow, expressed in a Cartesian coordinate system, is given as

$$
\begin{aligned}
\boldsymbol{\nabla} \cdot \boldsymbol{u} & =0 \\
\boldsymbol{\nabla} \cdot(\rho \boldsymbol{u} \otimes \boldsymbol{u}) & =-\nabla P+\boldsymbol{\nabla} \cdot \boldsymbol{\tau}
\end{aligned}
$$

where $\boldsymbol{\nabla} \equiv(\partial / \partial x, \partial / \partial y, \partial / \partial z)^{\mathrm{T}}, \boldsymbol{u} \equiv\left(u_{x}, u_{y}, u_{z}\right)^{\mathrm{T}}, \rho, P$ and $\boldsymbol{\tau}$ denote the gradient operator, velocity vector, fluid density, pressure and viscous stress tensor. The term $\tau$ can be expressed as

$$
\boldsymbol{\tau}=2 \mu \boldsymbol{D} ; \quad \boldsymbol{D}=\frac{1}{2}\left(\nabla \boldsymbol{u}+(\nabla \boldsymbol{u})^{\mathrm{T}}\right)
$$

where $\mu$ is the dynamic viscosity of the fluid and $\boldsymbol{D}$ is the rate-of-strain tensor. The Quemada model is employed to model the non-Newtonian blood behaviour due to its flexibility. It shows sufficient flexibility through its explicit coefficients that could account for various important elements affecting the blood viscosity [32]. Hence, the shear rate dependent viscosity is given by [33]

$$
\mu=\mu_{\mathrm{p}}\left[1-\frac{1}{2}\left(\frac{c_{0}+c_{\infty} \sqrt{\dot{\gamma} / \dot{\gamma}_{\text {crit }}}}{1+\sqrt{\dot{\gamma} / \dot{\gamma}_{\text {crit }}}}\right) H_{\mathrm{ct}}\right]^{-2} ; \quad \dot{\gamma}=\sqrt{2 \boldsymbol{D}: \boldsymbol{D}}
$$

where $\mu_{\mathrm{p}}$ is the blood plasma viscosity, $c_{0}$ is the rate of rouleaux formation at zero shear, $c_{\infty}$ is the rate of rouleaux formation at infinite shear, $H_{\mathrm{ct}}$ is the Haematocrit index, $\dot{\gamma}$ denotes the shear rate and $\dot{\gamma}_{\text {crit }}$ denotes the critical shear rate. The parameters in the Quemada model are extracted from the work of Marcinkowska-Gapińska et al. [32] where $\mu_{\mathrm{p}}=0.00128 \mathrm{~Pa} \cdot \mathrm{s}, c_{0}=4.01$, $c_{\infty}=1.77, H_{\mathrm{ct}}=0.45$ and $\dot{\gamma}_{\text {crit }}=4.2 \mathrm{~s}^{-1}$. 


\subsection{Governing equations for oxygenation}

In this work, the mathematical expression for the total amount of gas in blood is adopted from the work of Chan et al. [34]. The mathematical formulations for both $\mathrm{O}_{2}$ and $\mathrm{CO}_{2}$ are being expressed as physically dissolved and chemically bounded to haemoglobin. The total gas molar concentration is expressed as

$$
C_{i}=\alpha_{i} \mathscr{P}_{i}+H_{\mathrm{b}, i} S_{i}
$$

where $C$ is the gas molar concentration, $\alpha$ is the gas solubility, $\mathscr{P}$ is the partial pressure, $H_{\mathrm{b}}$ is molar concentration of gas that is bound to haemoglobin and $S$ is the gas saturation. The subscript $i$ denotes the gas species, which are $\mathrm{O}_{2}$ or $\mathrm{CO}_{2}$. With the assumption that the reaction of both gases with haemoglobin occurs instantaneously, the gas saturations are being described by two separate dissociation curves (Hill-type equation). Both dissociation curves are known to be a function of their opposing gas concentration, $\mathrm{pH}$, temperature and 2,3-diphosphoglycerate [35,36], which is not covered in the current scope of the work. Nevertheless, the Bohr and Haldane effects, whereby the $\mathrm{CO}_{2}$ affects the affinity of the $\mathrm{O}_{2}$ dissociation and vice versa respectively, are accounted in this current work and are written as

$$
S_{i}=\frac{\left(\mathscr{P}_{i} / \mathscr{P}_{i}^{0}\right)^{n_{i}}}{1+\left(\mathscr{P}_{i} / \mathscr{P}_{i}^{0}\right)^{n_{i}}} ; \quad \mathscr{P}_{i}^{0}=\beta_{1, i}\left(\frac{1+\beta_{2, i} \mathscr{P}_{j}}{1+\beta_{3, i} \mathscr{P}_{j}}\right)
$$

where their respective parameters are tabulated in Table 3. It should be noted that the subscript $j$ also refers to their respective gas species but in such a way that $i \neq j$. To model the oxygenation process in blood oxygenators, the molar concentration of $\mathrm{O}_{2}$ needs to be higher and $\mathrm{CO}_{2}$ to be lower at the fibre surface than the molar concentration in blood. Moreover, as blood flows past the surface of the fibres, a region is being developed due to the difference in concentration with the existence of concentration gradients. This developed region is known as the concentration boundary layer. The oxygenation process in blood is governed by the convection-diffusion equation [26,34], expressed as

$$
\boldsymbol{\nabla} \cdot\left(\boldsymbol{u} C_{i}\right)=\boldsymbol{\nabla} \cdot\left(D_{\mathrm{eff}, i} \boldsymbol{\nabla} C_{i}\right)+\boldsymbol{\nabla} \cdot\left[\left(D_{\mathrm{rbc}}-D_{\mathrm{eff}, i}\right) \boldsymbol{\nabla}\left(H_{\mathrm{b}} S\right)_{i}\right]
$$

where $D_{\text {eff }}$ and $D_{\text {rbc }}$ are the effective diffusivity of free $\mathrm{O}_{2}$ in blood and diffusivity of red blood cells respectively. Expanding Eqn. 5 into Eqn. 7, the resulting equation yields

$$
\boldsymbol{\nabla} \cdot\left[\boldsymbol{u}\left(\alpha \mathscr{P}+H_{\mathrm{b}} S\right)_{i}\right]=\boldsymbol{\nabla} \cdot\left(D_{\mathrm{eff}, i} \boldsymbol{\nabla}(\alpha \mathscr{P})_{i}\right)+\boldsymbol{\nabla} \cdot\left(D_{\mathrm{rbc}} \boldsymbol{\nabla}\left(H_{\mathrm{b}} S\right)_{i}\right)
$$




\begin{tabular}{|c|c|c|c|}
\hline Notation & Description & Value & Units \\
\hline$\alpha_{\mathrm{O}_{2}}$ & Solubility coefficient of $\mathrm{O}_{2}$ & $3.25 \times 10^{-5}$ & $\mathrm{~mL} \mathrm{O}_{2} / \mathrm{mL}$ blood $\mathrm{mmHg}$ \\
\hline$\alpha_{\mathrm{CO}_{2}}$ & Solubility coefficient of $\mathrm{CO}_{2}$ & $7.28 \times 10^{-4}$ & $\mathrm{~mL} \mathrm{CO}_{2} / \mathrm{mL}$ blood $\mathrm{mmHg}$ \\
\hline$H_{\mathrm{b}, \mathrm{O}_{2}}$ & Haemoglobin concentration in red blood cell & 0.2072 & $\mathrm{~mL} \mathrm{O}_{2} / \mathrm{mL}$ blood \\
\hline $\mathrm{H}_{\mathrm{b}, \mathrm{CO}_{2}}$ & Haemoglobin concentration of $\mathrm{CO}_{2}$ & 0.02692 & $\mathrm{~mL} \mathrm{CO}_{2} / \mathrm{mL}$ blood \\
\hline$n_{\mathrm{O}_{2}}$ & Hill exponent for $\mathrm{O}_{2}$ & 2.6069 & - \\
\hline$n_{\mathrm{CO}_{2}}$ & Hill exponent for $\mathrm{CO}_{2}$ & 0.5497 & - \\
\hline$\beta_{1, \mathrm{O}_{2}}$ & Hill parameter 1 for $\mathrm{O}_{2}$ & 14.99 & $\mathrm{mmHg}$ \\
\hline$\beta_{2, \mathrm{O}_{2}}$ & Hill parameter 2 for $\mathrm{O}_{2}$ & 0.03198 & $\mathrm{mmHg}^{-1}$ \\
\hline$\beta_{3, \mathrm{O}_{2}}$ & Hill parameter 3 for $\mathrm{O}_{2}$ & 0.008275 & $\mathrm{mmHg}^{-1}$ \\
\hline$\beta_{1, \mathrm{CO}_{2}}$ & Hill parameter 1 for $\mathrm{CO}_{2}$ & 194.4 & $\mathrm{mmHg}$ \\
\hline$\beta_{2, \mathrm{CO}_{2}}$ & Hill parameter 2 for $\mathrm{CO}_{2}$ & 0.05591 & $\mathrm{mmHg}^{-1}$ \\
\hline$\beta_{3, \mathrm{CO}_{2}}$ & Hill parameter 3 for $\mathrm{CO}_{2}$ & 0.03255 & $\mathrm{mmHg}^{-1}$ \\
\hline$D_{\text {eff }, \mathrm{O}_{2}}$ & Effective diffusion coefficient for $\mathrm{O}_{2}$ in blood & $2.24 \times 10^{-9}$ & $\mathrm{~m}^{2} / \mathrm{s}$ \\
\hline$D_{\text {eff }, \mathrm{CO}_{2}}$ & Effective diffusion coefficient for $\mathrm{CO}_{2}$ in blood & $1.25 \times 10^{-9}$ & $\mathrm{~m}^{2} / \mathrm{s}$ \\
\hline$D_{\mathrm{rbc}}$ & Diffusion coefficient of red blood cells & $1.50 \times 10^{-11}$ & $\mathrm{~m}^{2} / \mathrm{s}$ \\
\hline
\end{tabular}

Table 3. Gas transport parameters for blood. The concentration unit can be converted from $\mathrm{mL} / \mathrm{mL}$ to $\mathrm{mol} / \mathrm{L}$ by a factor of 0.045 .

The term on the left represents the convection of dissolved gas species and bounded to haemoglobin. The terms on the right hand side are interpreted as the diffusion of dissolved gas species and the diffusion of red blood cells to allow for the formation of oxy-haemoglobin, respectively in blood. When $H_{\mathrm{b}}=0$, Eqn. 8 will therefore represent a gas transport equation of a fluid without the presence of haemoglobin.

\subsection{Oxygenation transfer evaluation}

The overall oxygenation performance in a blood oxygenator is evaluated based on an important parameter known as the overall mass transfer coefficient [3]. This assessment can be computed through the gas transfer rate $(\dot{V})$ which is expressed as

$$
\dot{V}_{i}=k_{i} A \Delta C_{i}
$$

where $k$ denotes the overall mass transfer coefficient, $A$ represents the total fibre membrane area and $\Delta C$ is overall concentration difference between fibre surface and blood. The overall mass transfer coefficient can alternatively be known as the overall mass transfer resistance and has contributions from the mass transfer coefficients in blood $\left(k_{\mathrm{b}}\right)$, across the membrane $\left(k_{\mathrm{m}}\right)$ and inside the fibre $\left(k_{\mathrm{g}}\right)$. It was reported that the influence of both $k_{\mathrm{g}}$ [29] and $k_{\mathrm{m}}$ [3] were negligible in the calculation of the overall mass transfer coefficient and therefore $k \approx k_{\mathrm{b}}$. The overall mass transfer coefficient is commonly obtained through experiments for an entire device [7-9]. However, in this current work, the computation of $k$ is based on a representative fibre and is taken as the surface averaged local mass transfer coefficient which involves combining expressions from Fick's law 
and an analogous equation similar to Newton's law of cooling for mass transfer $[37,38]$ which can be written as

$$
k_{i} \equiv \bar{k}_{i}=\frac{1}{2 \pi} \int_{0}^{2 \pi} k_{\mathrm{L}, i} d \theta ; \quad k_{\mathrm{L}, i}=-\frac{\left.D_{\mathrm{eff}, i}\left(\boldsymbol{n} \cdot \nabla C_{i}\right)\right|_{\mathrm{f}}}{\left(C_{\mathrm{f}, i}-C_{\mathrm{in}, i}\right)}
$$

where $\boldsymbol{n}$ is the unit vector normal to the fibre surface and the subscripts ' $\mathrm{f}$ ' and 'in' refer to the fibre surface and inlet respectively.

A more common way of expressing the mass transfer coefficient is through the introduction of a dimensionless parameter known as the Sherwood number $\left(S h_{i}=k_{i} d / D_{\text {eff }, i}\right)$. The advantage of this method is that it allows large data containing different fluid velocities and fibre configurations to be incorporated through the mass transfer correlation expressed in the general form of $S h=a R e^{b} S c^{0.33}$ for transverse and parallel flows. This correlation is usually established through experiments but the current work the correlation are constructed via post-processing of the numerical simulations. Based on the simulation results, the local Sherwood number $\left(S h_{\mathrm{L}}\right)$ on a fibre surface is initially evaluated and can be written as

$$
S h_{\mathrm{L}, i} \equiv \frac{k_{\mathrm{L}, i} d}{D_{\mathrm{eff}, i}}=\frac{\left.2 r \alpha_{i}\left(\boldsymbol{n} \cdot \nabla \mathscr{P}_{i}\right)\right|_{\mathrm{f}}}{\left(C_{\mathrm{f}, i}-C_{\mathrm{in}, i}\right)}
$$

where $r$ denotes the fibre radius. In order to assess the overall gas contribution, the surface averaged Sherwood number $(\overline{S h})$ along a fibre surface for $\mathrm{O}_{2}$ and $\mathrm{CO}_{2}$ can be expressed as

$$
\overline{S h}_{i}=\frac{1}{2 \pi} \int_{0}^{2 \pi} S h_{\mathrm{L}, i} d \theta
$$

The computed $S h$ will then be treated as a basis to construct mass transfer correlations, including various fibre configurations and flow directions, that could be used at the oxygenator scale. The computed $S h$ is initially rearranged and plotted against $R e$ where they are presented in a bi-logarithmic format. Their respective parameters $a$ and $b$, from the mass transfer correlation $S h=a R e^{b} S c^{0.33}$, are obtained via least squares, for various porosities, flow directions and fibre configurations. These mass transfer correlations are then further generalised by investigating the dependence of these mass transfer correlations on geometrical and flow features.

\subsection{Numerical setup}

Transverse flow simulations are performed two-dimensionally (2D). Based on the recommendations from the work of Chan et al. [27] and Zierenberg et al. [28], the domain will consist of 20 unit cells linked together along the flow direction. This is to account for the possible impact of gas transfer in one fibre induced towards the adjacent fibres [28]. In contrast, a unit cell is only considered for parallel flow simulations which are conducted in three-dimensions (3D). The domain is 
extended to a millimeter thick to allow flow to be fully developed. The computational grid for the transverse flow simulations consists of unstructured hexahedrons with the density being increased exponentially towards the fibre surface in order to fully capture the velocity and gas concentration gradients. The computational grid for parallel flow simulation has an extrusion of structured hexahedrons adopted from the transverse flow grid.

Boundary conditions for the governing equations of fluid flow involve a uniform velocity that is prescribed at the inlet and a pressure of zero at the outlet. An averaged velocity $(\bar{u})$ is initially computed and the prescribed inlet velocity is being scaled depending on $\varepsilon$ in order to consistently maintain $\operatorname{Re}(=\rho \bar{u} d / \mu)$. For transverse flow, the velocity is scaled based on the ratio of spacing over the gap. The scaled inlet velocity for parallel flow is being divided by its porosity. This scaled velocity is known as the superficial velocity. Furthermore, no-slip boundary conditions are imposed along the fibre walls and symmetric boundary conditions are imposed on other undefined regions. Next, boundary conditions for gas transport involved prescribing an inlet venous partial pressure of $40 \mathrm{mmHg}$ and $45 \mathrm{mmHg}$ for $\mathrm{O}_{2}$ and $\mathrm{CO}_{2}$ respectively based on normal adult [39]. Pure $\mathrm{O}_{2}$ partial pressure of $760 \mathrm{mmHg}$ and $\mathrm{CO}_{2}$ partial pressure of $0 \mathrm{mmHg}$ are prescribed in all fibre walls. The values of Schmidt number $\left(\mathrm{Sc}\right.$ ) are computed to be approximately 1,467 for $\mathrm{O}_{2}$ and 2,738 for $\mathrm{CO}_{2}$ based on blood density being $1,050 \mathrm{~kg} / \mathrm{m}^{3}$ and a constant viscosity at high shear rate range of $0.00345 \mathrm{~Pa} \cdot \mathrm{s}$.

The computer-aided design model geometry is generated using SolidWorks ${ }^{\circledast}$ and imported into the Meshing module of ANSYS Workbench v14.5 [40] to perform spatial discretisation. The steady state simulation is performed using ANSYS Fluent ${ }^{\circledast}[40]$. The software utilises a cell-centred finite volume method. The governing equations are discretised using a second order upwind scheme and coupling between velocity and pressure is achieved by using the standard PISO algorithm. For blood simulations, the Quemada model is written as a user-defined function (UDF). The steady state continuity and momentum equations (Eqns. 1 and 2) are solved iteratively until the respective scaled residuals of $10^{-10}$ are achieved. The converged steady state velocity fields are then used as a basis for solving the convection-diffusion equations for $\mathrm{O}_{2}$ and $\mathrm{CO}_{2}$ (Eqn. 8) iteratively until a similar convergence criterion is achieved. ANSYS CFD-Post ${ }^{\boxplus}$ is used for post-processing purposes.

A mesh sensitivity study is conducted for transverse flow setup by comparing the computed pressure drop, maximum velocity and $\mathrm{Sh}$ for $\mathrm{O}_{2}$ and $\mathrm{CO}_{2}$ from the $19^{\text {th }}$ fibre using a square fibre arrangement of porosity 0.7 and 0.3 at $R e=10$. The relative error, $e$ for each variable is defined as

$$
e_{\psi_{M_{i}}}=\frac{\left|\psi_{M_{i}}-\psi_{N}\right|}{\psi_{N}} ; \quad i \in\{1,2, \ldots N-1\}
$$

where $\psi$ is the variable, subscripts $i$ and $N$ denote the mesh number and finest mesh respectively. The mesh convergence study for both porosities are tabulated in Table 4. It can be seen that all four monitoring variables decrease when the number of elements increases. Thereafter, all the simulations are based on mesh M4, which ensures a good precision at a reasonable computational cost. Next, the porosities and their respective mesh sizes used in the transverse flow mesh sensitivity study is adopted for parallel flow. Again, the computed pressure drop, maximum velocity and $S h$ for $\mathrm{O}_{2}$ and $\mathrm{CO}_{2}$ are monitored for 


\begin{tabular}{l|rrrrr|rrrrr}
\hline \hline & \multicolumn{5}{|c|}{ Porosity 0.7 } & \multicolumn{5}{c}{ Porosity 0.3 } \\
\hline Meshes & $\mathrm{M} 1$ & $\mathrm{M} 2$ & $\mathrm{M} 3$ & $\mathrm{M} 4$ & $\mathrm{M} 5$ & $\mathrm{M} 1$ & $\mathrm{M} 2$ & $\mathrm{M} 3$ & $\mathrm{M} 4$ & $\mathrm{M} 5$ \\
\hline Elements & 942 & 3,582 & 7,954 & 14,720 & 34,516 & 1,043 & 2,714 & 5,605 & 21,175 & 39,773 \\
$e_{\Delta \mathrm{P}}$ & 0.0036 & 0.0032 & 0.0029 & 0.0018 & - & 0.0385 & 0.0171 & 0.0073 & 0.0001 & - \\
$e_{\|\boldsymbol{u}\| \max }$ & 0.0073 & 0.0016 & 0.0006 & 0.0003 & - & 0.0592 & 0.0299 & 0.0147 & 0.0026 & - \\
$e_{\mathrm{Sh}}$ & 0.4023 & 0.0908 & 0.0218 & 0.0109 & - & 0.7339 & 0.2105 & 0.1204 & 0.0182 & - \\
$e_{\mathrm{Sh}_{\mathrm{CO}}}$ & 0.3246 & 0.0624 & 0.0065 & 0.0019 & - & 0.5258 & 0.1011 & 0.0075 & 0.0071 & - \\
\hline \hline
\end{tabular}

Table 4. Transverse flow mesh sensitivity study for square arranged fibres of porosity 0.7 and 0.3 at $\operatorname{Re}=10$. Note that the number of elements represents a unit cell.

\begin{tabular}{l|rrrr|rrrr}
\hline \hline & \multicolumn{3}{|c|}{ Porosity 0.7 } & \multicolumn{4}{c}{ Porosity 0.3 } \\
\hline Meshes & $\mathrm{M} 1$ & $\mathrm{M} 2$ & $\mathrm{M} 3$ & $\mathrm{M} 4$ & $\mathrm{M} 1$ & $\mathrm{M} 2$ & $\mathrm{M} 3$ & $\mathrm{M} 4$ \\
\hline Elements & 180,400 & 360,800 & 721,600 & $1,443,200$ & 312,325 & 624,650 & $1,249,300$ & $2,498,600$ \\
$e_{\Delta \mathrm{P}}$ & 0.1192 & 0.0777 & 0.0210 & - & 0.0748 & 0.0434 & 0.0188 & - \\
$e_{\|\boldsymbol{u}\| \max }$ & 0.0051 & 0.0041 & 0.0031 & - & 0.0080 & 0.0070 & 0.0050 & - \\
$e_{\mathrm{Sh}_{\mathrm{O}_{2}}}$ & 0.0147 & 0.0123 & 0.0017 & - & 0.0034 & 0.0024 & 0.0007 & - \\
$e_{\mathrm{Sh}_{\mathrm{CO}_{2}}}$ & 0.0159 & 0.0100 & 0.0079 & - & 0.0132 & 0.0050 & 0.0006 & - \\
\hline \hline
\end{tabular}

Table 5. Parallel flow mesh sensitivity study for square arranged fibres of porosity 0.7 and 0.3 at $R e=10$.

mesh convergence and their errors are tabulated in Table 5. Based on this, it can be seen that mesh M3 can be considered to be converged as the relative errors are below $2 \%$. Mesh sensitivity studies of transverse and parallel flows were not performed for staggered arrangement. However, mesh size is kept consistent with the chosen mesh used for square arrangement to ensure good accuracy.

\section{Results and Discussions}

\subsection{Numerical validation}

A numerical validation is performed using Newtonian fluid with two objectives. Firstly, it is to ensure that the simulation results can reproduce findings from the literature for a wide range of $\varepsilon$, flow direction and fibre arrangement. Secondly, it is to ensure that the $R e$ range studied in this investigation is in the low Re regime (Stokes regime) in which Darcy's equation is valid, where the relationship between the volume-averaged velocity through a porous media and the pressure drop is linear. Newtonian blood is being considered which has a density of $1050 \mathrm{~kg} / \mathrm{m}^{3}$ and a dynamic viscosity of $0.00345 \mathrm{~Pa} \cdot \mathrm{s}$ [41]. The results obtained are validated against analytical formulations from Gebart [42] and Tamayol and Bahrami [43] for transverse and parallel flow respectively. The non-dimensional transverse flow permeability $\left(K_{\perp}^{*} \equiv K_{\perp} / d^{2}\right)$ and parallel flow permeability $\left(K_{\|}^{*} \equiv K_{\|} / d^{2}\right)$ under $R e=0.5$ and $R e=10$ for square and staggered configurations are plotted in Figures 2(a) and 2(b) respectively. According to both figures, it can be observed that the numerical results provide excellent correlation with the analytical formulations. This also proves that the $R e$ used in this investigation is performed under the consideration of minimal or no inertia effects which is within the range reported by Dierickx et al. [22] showing a value of 9.2 and Coulaud et al. [44] reporting the value of 13 . 


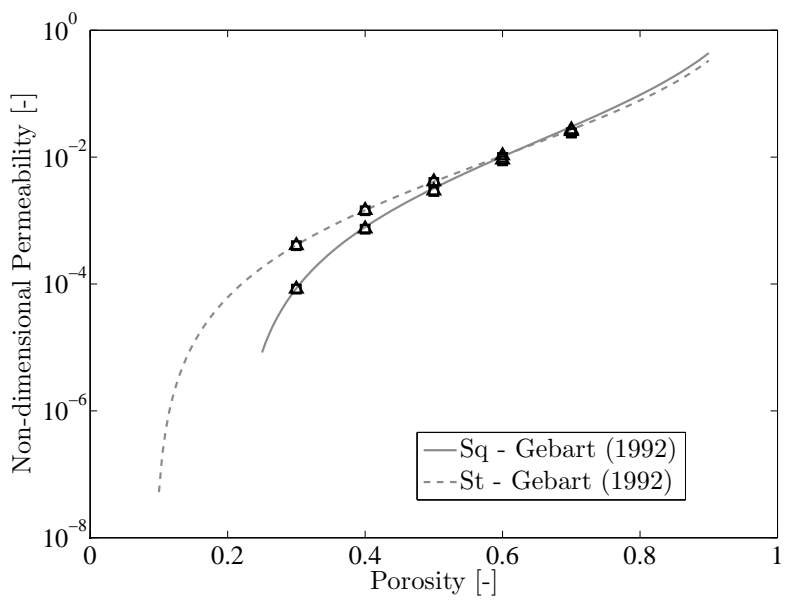

(a) Transverse Flow

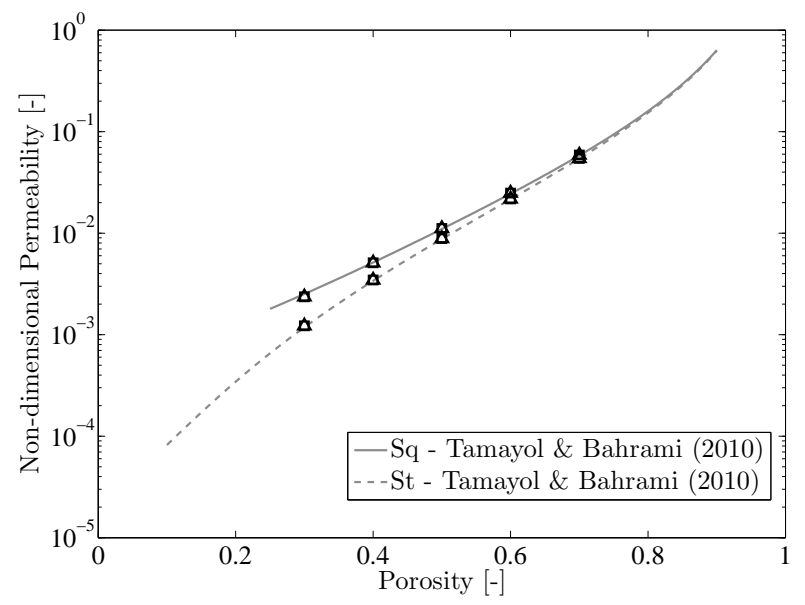

(b) Parallel Flow

Fig. 2. Comparison of non-dimensional permeability for square and staggered configurations due to (a) transverse and (b) parallel flows against analytical results based on $R e=0.5(\triangle)$ and $R e=10(\square)$. Numerical results are indicated as black and blue for square and staggered configurations respectively. Analytical expression abbreviations is indicated as (Sq) and (St) for square and staggered configurations respectively.

\subsection{Concentration development analysis}

It is known that the respective concentration will gradually increase (for the case of $\mathrm{O}_{2}$ ) or decrease (for the case of $\mathrm{CO}_{2}$ ) while travelling through the domain. In other words, the concentration gradients in the vicinity of the fibre surface will gradually decrease until blood is fully oxygenated (zero concentration gradients). This spatial decreasing trend directly affects the mass transfer coefficient/Sherwood number, making it difficult to establish mass transfer correlations for $\mathrm{O}_{2}$ and $\mathrm{CO}_{2}$. Therefore, the current section aims to investigate the development of $\mathrm{O}_{2}$ and $\mathrm{CO}_{2}$ concentration fields in blood across and along fibre(s) whereby a representable mass transfer coefficient/Sherwood number for each gas species is sought after to describe the overall effects.

This investigation utilises the computational domain described in Section 2.4 for transverse and parallel flows alongside with their respective boundary conditions. The surface average Sherwood number, $\overline{S h}$, of each fibre is being monitored. Figures 3(a) and 3(b) illustrate the change in $\overline{S h}_{O_{2}}$ and $\overline{S h}_{\mathrm{CO}_{2}}$ across (for transvers flow) and along (for paralle flow) the fibre(s), respectively. Furthermore, the normalised concentrations are computed $\left(C^{*} \equiv \bar{C}_{i} / \bar{C}_{\mathrm{in}, i}\right)$ where the gradual increasing and decreasing for $\mathrm{O}_{2}$ and $\mathrm{CO}_{2}$ trends are seen, respectively. It can be observed that the $\overline{S h}$ for both $\mathrm{O}_{2}$ and $\mathrm{CO}_{2}$ decreases dramatically at the beginning and slowly approaches to a constant decreasing magnitude. Provided that the domain is infinitely long, this trend will progressively head towards equilibrium where the prescribed values at the fibre surface are achieved. Therefore, expressions for $\overline{S h}$ can then be established via least squares for each gas species, porosity and flow direction, covering the range from a typical deoxygenated blood up to a fully oxygenated blood condition. The expressions are found to be in the form of power law functions, $\overline{S h}_{\perp, i}=\phi N_{\mathrm{f}}^{-\xi}$ and $\overline{S h}_{\|, i}=\phi z^{-\xi}$ for transverse and parallel flows, respectively. Their respective fitted parameters are tabulated in Appendix (Section 6).

The next step is crucial to obtain a representable $\overline{S h}$ describing a specific blood oxygenator. A cut-off point, in terms of fibre numbers or length of fibre, for each flow direction function is introduced to avoid the under-estimation and over- 
estimation of $\overline{S h}$. In this work, we have adopted typical commercial oxygenators presented in the work of Wickramasinghe et al. [7] and Dierickx et al. [13] where the fibres approximately 11,000 to 15,000 in total with a length ranging from 12.4 $\mathrm{cm}$ and $20 \mathrm{~cm}$. In order to identify a suitable estimation for $\overline{\mathrm{Sh}}$, we have taken values in between these range and they are 120 fibres across one direction (assuming a square device) with a length of $15 \mathrm{~cm}$. A further averaging procedure is performed for each flow direction to obtain this representable Sherwood number and is written as

$$
S h_{\perp, i}=\frac{1}{N_{\mathrm{f}}} \int_{1}^{N_{\mathrm{f}}} \overline{S h}_{i} d N \quad \text { for transverse flow; } \quad S h_{\|, i}=\frac{1}{L_{\mathrm{f}}} \int_{0}^{L_{\mathrm{f}}} \overline{S h_{i}} d z \quad \text { for parallel flow; }
$$

where $N_{\mathrm{f}}$ denotes the maximum number of fibres that is perpendicular to the flow direction and $L_{\mathrm{f}}$ is the length of fibre. The computed $S h_{\perp, i}$ is found to be consistenty situated on the 42nd fibre for transverse flow based on several tests which include various porosity and $R e$. As for parallel flow, the computed $S h_{\|, i}$ is found to be $5 \mathrm{~cm}$ in length. They will be used throughout the remainder of this work as a guidance for the construction of mass transfer correlations of $\mathrm{O}_{2}$ and $\mathrm{CO}_{2}$ at various fibre porosities, $R e$ and flow directions. Therefore, this section is deemed to be an important procedure prior to establishing any mass transfer correlation for the device of interest.

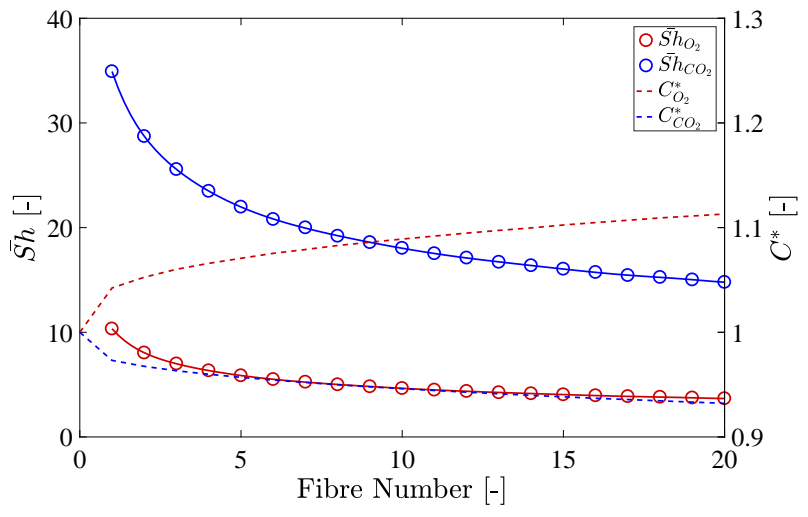

(a) Transverse Flow

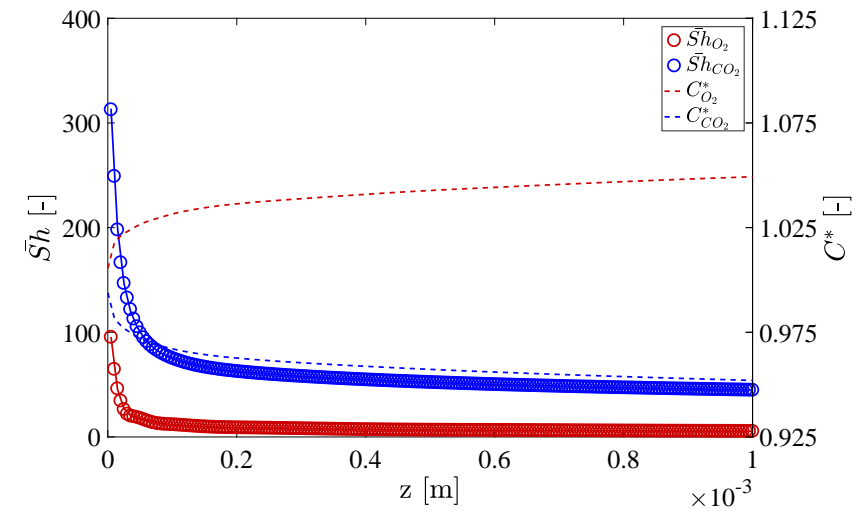

(b) Parallel Flow

Fig. 3. The development of $\overline{S h}_{O_{2}}$ and $\overline{S h}_{C O_{2}}$ for square configurations of (a) transverse and (b) parallel flows based on $\varepsilon=0.7$ at $R e=10$. The solid lines represent fitted data via least squares with $\mathrm{R}^{2}$ correlations of 0.95 and above. The dash lines refer to their respective $C^{*}$.

\subsection{Blood flow and gas transport behaviour analysis}

In this section, the results obtained from the set of simulations of square and staggered fibre arrangements are discussed separately for transverse and parallel flow. Contour plots between the $18^{\text {th }}$ and $19^{\text {th }}$ for transverse flow and $0.9 L$ for parallel flow are presented to illustrate typical flow, $\mathrm{O}_{2}$ and $\mathrm{CO}_{2}$ distributions. The analysis includes a description on the blood flow behaviour where the velocity and shear stress distributions are discussed. This is to identify regions of recirculation and high shear stresses, computed as $\tau=\mu \dot{\gamma}$, as they are linked to the potential occurrence of haemolysis [45] and thrombosis [46,47]. 
The likelihood of blood trauma is higher in regions where blood is exposed to higher shear, with the threshold reported to be $150 \mathrm{~Pa}[48,49]$. Furthermore, regions with shear stress greater than $50 \mathrm{~Pa}$ is commonly used an indicator of potential platelet activation which could lead to thrombus formation [46,49]. Next, $\mathrm{O}_{2}$ and $\mathrm{CO}_{2}$ transport results are discussed to provide an insight into the oxygenation process with its local gas transfer efficiency. The establishment of mass transfer correlations, based on the aforementioned method in Section 3.2, are presented at the end, which can be used to simulate flow through specific oxygenator designs. These correlations are quantified via least squares with an $\mathrm{R}^{2}$ correlation. Furthermore, we sought to generalise the fitted correlations through the parameters $a$ and $b$ of the general mass transfer correlation of $S h=a R e^{b} S c^{0.33}$ for both transverse and parallel flow as much as possible to provide a wider set of applications. The decision to adopt a similar mass transfer correlation for parallel flow is due to the low Reynolds number range of this investigation. The introduction of the additional term $d_{h} / L$ is to take into account the development of concentration profiles down the fibre [50] which is neglected in this current work due to the low Reynolds numbers typical for blood oxygenators.

\subsubsection{Transverse Flow Analysis}

Flow: Figures 4(a) to 4(h) depict the velocity magnitude contour, normalised by its respective maximum velocity magnitude, and shear stress contour for both configurations, respectively at the top and bottom halves of the unit cell. According to the results, high magnitude in fluid velocity and shear stress regions are intuitively observed in between the narrow gaps of the wall. Furthermore, both magnitudes increase as $\varepsilon$ reduces.

Streamlines are superimposed onto the normalised velocity magnitude contours to identify regions of recirculation as they could potentially initiate the formation of blood clots [51], especially in sufficiently small gap regions, and also affect the overall mass transfer performance. At $R e=0.5$, corner eddies are first observed close to the fibres in the square configuration of $\varepsilon=0.7$, as depicted in Figure 4(a). The size of those two corner eddies will start to increase as the gap decreases until they merge to form one central eddy. The shape of the central eddy will continue to alter as the gap is further decreased up to a point where two additional counter rotating eddies appear close to the fibres. These two additional eddies will subsequently form into one eddy, rotating in the opposite direction with the adjacent eddy, as shown in Figure 4(b). The flow separation occurrence phenomena in the staggered arrangement is slightly delayed compared to the square arrangement due to its tortuous flow path (Figures 4(c) and 4(d)). Figures 4(i) to 4(1) illustrate circumferential shear stress profile of a fibre. Highest wall shear stress regions are observed at the centre along the circumference of the fibre wall as illustrated in Figures 4(i) and 4(j). In staggered configurations, a high shear stress region is observed at $60^{\circ}$ and $120^{\circ}$ (see Figures $4(\mathrm{k})$ and $4(\mathrm{l})$ ).

As $R e$ increases, the size and intensity of the recirculation grows and is subsequently skewed towards the upstream direction for both configurations as flow accelerates. The central point of the recirculation will initially remain at the centre and starts to shift towards the upstream fibre, illustrated in Figures 4(e) and 4(f). Similar trends can be observed for staggered arrangements when $R e$ is increased in which the occurrence of flow separation is slightly delayed as illustrated in Figures 4(g) and 4(h), respectively. The peak shear stress tends to shift towards upstream at $\varepsilon=0.7$ as shown in Figure 4(i) but becomes less obvious as $\varepsilon$ decreases due to the restrictive gaps in between fibres illustrated in Figure 4(j). Again, similar trends are observed for wall shear stress in staggered arrangements as depicted in Figures 4(k) and 4(l). 


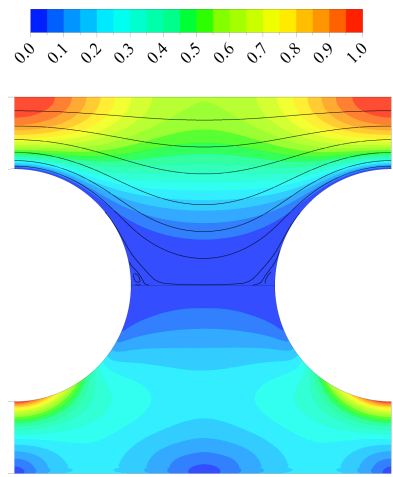

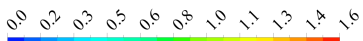

(a) $\mathrm{Sq} ; \varepsilon=0.7 ; \operatorname{Re}=0.5$
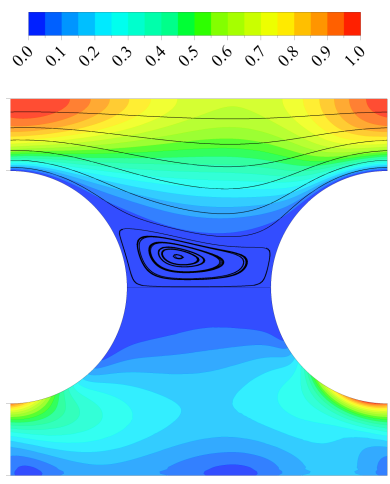

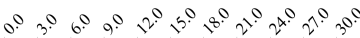

(e) $\mathrm{Sq} ; \varepsilon=0.7 ; \operatorname{Re}=10$

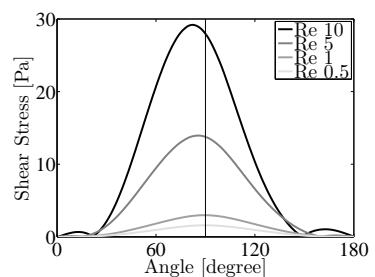

(i) $\mathrm{Sq} ; \varepsilon=0.7$
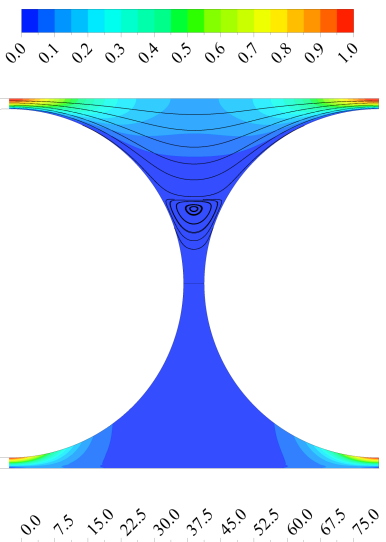

(b) $\mathrm{Sq} ; \varepsilon=0.3 ; \operatorname{Re}=0.5$
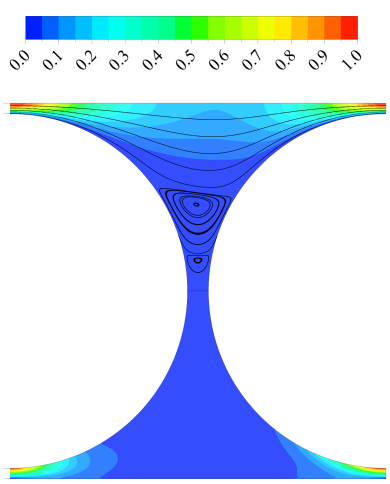

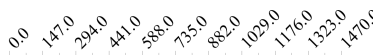

(f) $\mathrm{Sq} ; \varepsilon=0.3 ; \operatorname{Re}=10$

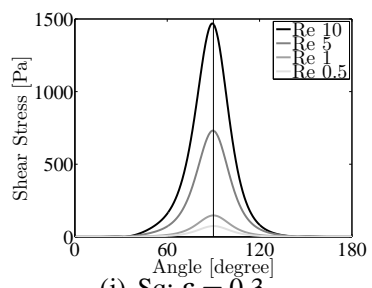

(j) $\mathrm{Sq} ; \varepsilon=0.3$

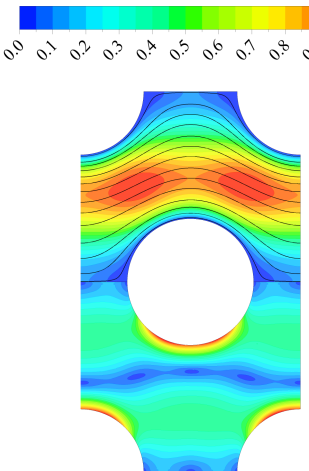

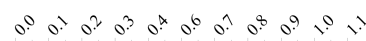

(c) $\mathrm{St} ; \varepsilon=0.7 ; R e=0.5$
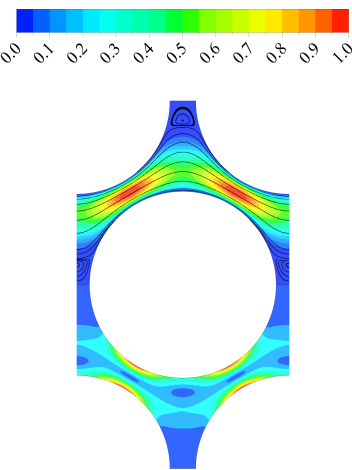

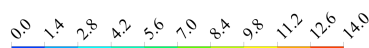

(d) $\mathrm{St} ; \varepsilon=0.3 ; \operatorname{Re}=0.5$

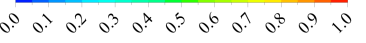

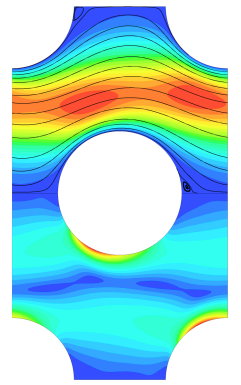

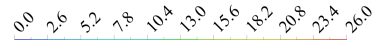

(g) St; $\varepsilon=0.7 ; R e=10$

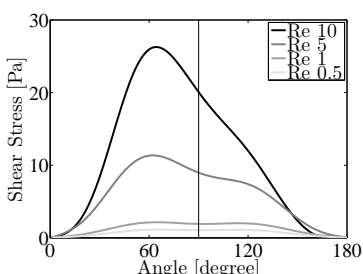

(k) $\mathrm{St} ; \varepsilon=0.7$

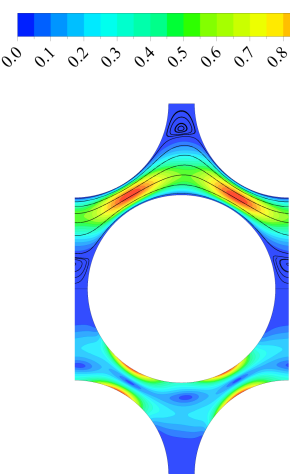

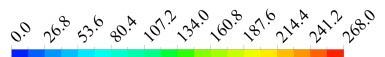

(h) $\mathrm{St} ; \varepsilon=0.3 ; \operatorname{Re}=10$

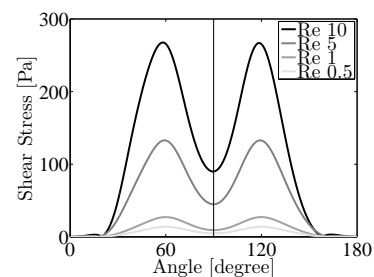

(1) $\mathrm{St} ; \varepsilon=0.3$

Fig. 4. Normalised velocity magnitude with streamlines superimposed (top half) and shear stress contours (bottom half) of porosity 0.7 and 0.3 for transverse flow past square $(\mathrm{Sq})$ and staggered $(\mathrm{St})$ configurations at $(\mathrm{a}-\mathrm{d}) \operatorname{Re}=0.5$ and $(\mathrm{e}-\mathrm{h}) \operatorname{Re}=10$. Contour legend in the top and bottom depicts the normalised velocity magnitude and shear stress respectively. ( $\mathrm{i}-\mathrm{l}$ ) Circumferential wall shear stress along one fibre of porosity 0.7 and 0.3 at (a) $R e=0.5$ and $(\mathrm{b}-\mathrm{c}) 10$ transverse flow past square (Sq) and staggered (St) configurations.

Oxygenation: Figures 5(a) to 5(h) depict both partial pressures of $\mathrm{O}_{2}$ and $\mathrm{CO}_{2}$, computed using Eqn. 11 normalised with their respective initial partial pressures, for transverse flow past square and staggered configurations respectively. The contours are displayed separately at the top and bottom halves of the domain for $\mathrm{O}_{2}$ and $\mathrm{CO}_{2}$.

It can be observed that the partial pressure boundary layers for $\mathrm{O}_{2}$ and $\mathrm{CO}_{2}$ appeared to be thicker when $R e$ is low, as illustrated in Figure 5(a) for $\varepsilon=0.7$ at $R e=0.5$. A thicker boundary layer (low partial pressure gradient) leads to low mass 
transfer coefficient and hence less efficient gas transfer at the fibre surface. This can be improved as $\varepsilon$ decreases as the volume of blood to be oxygenated is reduced, as depicted in Figure 5(b). Similar trends are applied to staggered configurations in Figures 5(c) and 5(d).

Figures 5(i) to 5(l) illustrate the circumferential $S h_{\mathrm{L}, \mathrm{O}_{2}}$ and $S h_{\mathrm{L}, \mathrm{CO}_{2}}$ profiles of one fibre. The profiles for $S h_{\mathrm{L}, \mathrm{O}_{2}}$ is smaller than $\mathrm{Sh}_{\mathrm{L}, \mathrm{CO}_{2}}$ due to the higher solubility of $\mathrm{CO}_{2}$ (at least an order of magnitude higher). At $R e=0.5$, the highest $S h_{\mathrm{L}, \mathrm{O}_{2}}$ and $S h_{\mathrm{L}, \mathrm{CO}_{2}}$ are observed at the center of the fibre (smaller gap regions) where velocity gradient is high leading to high partial pressure gradient and gas transfer. The upstream and downstream $S h_{\mathrm{L}}$ is not zero which indicates gas transfer activities due to the corner recirculating eddies, as illustrated in Figure 5(i). However, $S h_{\mathrm{L}, \mathrm{O}_{2}}$ and $S h_{\mathrm{L}, \mathrm{CO}_{2}}$ tend to reduce to zero as $\varepsilon$ decreases, suggesting that there is a possibility of stagnation region where no gas transfer occurs, see Figure $5(\mathrm{j})$. For staggered arrangement, the shape of the profile is caused by its geometrical nature where flow is less restrictive for $\varepsilon$ of 0.7 and vice versa as $\varepsilon$ decreases where two peaks of similar magnitude is visible. Furthermore, the upstream $S h_{\mathrm{L}, \mathrm{O}_{2}}$ and $S h_{\mathrm{L}, \mathrm{CO}_{2}}$ are seen to be higher compared to the ones downstream, as depicted in Figure 5(k). However, their values tend to be equal and reducing to zero as $\varepsilon$ decreases, see Figure 5(1).

As $R e$ increases, the partial pressure boundary layer is observed to be thinner (higher partial pressure gradient) leading to higher mass transfer coefficient and as a result more efficient in gas transfer at the fibre surface, see Figures 5(e) and 5(f). When the inertial forces in the flow are large enough, the boundary layer of gas partial pressure for both $\mathrm{O}_{2}$ and $\mathrm{CO}_{2}$ starts to break off from the fibre surface due to flow separation towards the main flow, forming a tail-like filament attaching along the recirculation region. Similar trends are applied to staggered configurations in Figures 5(g) and 5(h).

The circumferential $S h_{\mathrm{L}, \mathrm{O}_{2}}$ and $S h_{\mathrm{L}, \mathrm{CO}_{2}}$ profiles are higher and shifted towards the upstream region of the fibre. Their magnitude increases as $\varepsilon$ decreases. At low $\varepsilon$, the bulk of the fluid tends to flow tangentially to the fibre gaps, causing less blood to be in contact with the fibre, hence reducing $S h_{\mathrm{L}, \mathrm{O}_{2}}$ and $S h_{\mathrm{L}, \mathrm{CO}_{2}}$ and forcing the profile to be steeper at the center of the fibre. A secondary peak of smaller magnitude is observed between $140^{\circ}$ and $160^{\circ}$ in both $S h_{\mathrm{L}}$ profiles and is caused by the recirculating flow. The magnitude of this secondary peak depends heavily on the intensity of the recirculation zone. For a staggered arrangement, the differences between the upstream and downstream $S h_{\mathrm{L}}$ are observed to be larger. The secondary peak is observed between $160^{\circ}$ and $170^{\circ}$.

Mass transfer correlation: To compare the overall oxygenation for varying $\varepsilon$, flow regime and fibre configuration, it is useful to consider the averaged Sherwood number as computed through Eqn 12. Figures 6(a) and 6(b) depict the least squares fitted mass transfer correlations of $\mathrm{O}_{2}$ and $\mathrm{CO}_{2}$ at various porosities for both square and staggered configurations. Each point on the graph represents the average Sherwood number and is plotted as a function of $R e$ in a bi-logarithmic format. According to the results, it is shown that all the mass transfer correlations at each $\varepsilon$ and fibre arrangement can be fitted as a linear correlation, in logarithmic form, with an $\mathrm{R}^{2}$ correlation of 0.99 and above. The power laws used to fit through the data points can be found in Table 6 for square and staggered configurations. It can be observed that the fitted mass transfer correlation expressions for both $\mathrm{O}_{2}$ and $\mathrm{CO}_{2}$ are vastly different and no common ground could be found hence it did not seem possible to generalise the two gas types into one expression. However, a further generalisation can be achieved by 


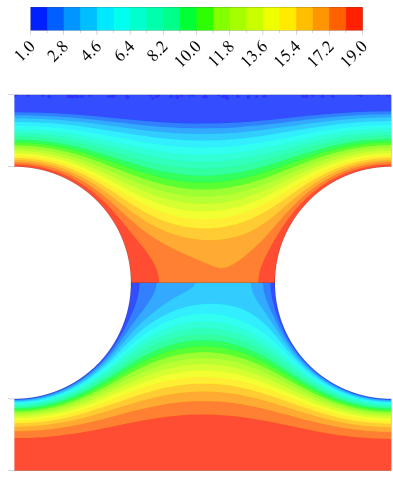

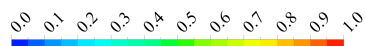

(a) Sq. $\varepsilon=0.7 ; \operatorname{Re}=0.5$
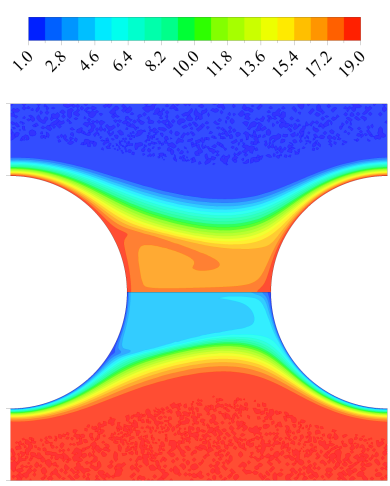

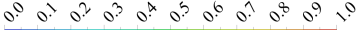

(e) Sq. $\varepsilon=0.7 ; \operatorname{Re}=10$

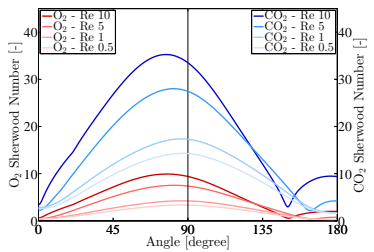

(i) $\mathrm{Sq} . \varepsilon=0.7$

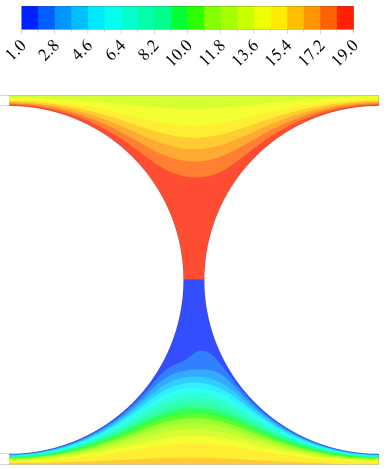

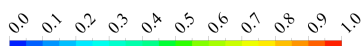

(b) Sq. $\varepsilon=0.3 ; R e=0.5$

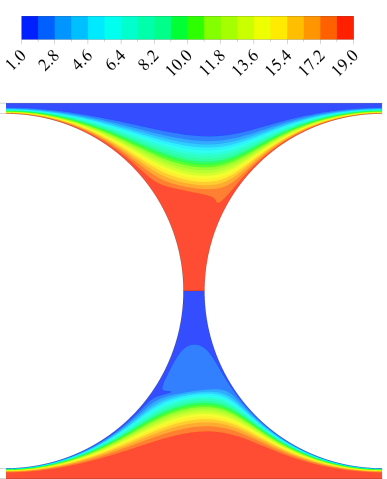

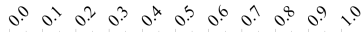

(f) Sq. $\varepsilon=0.3 ; R e=10$

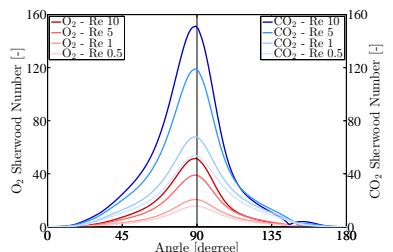

(j) $\mathrm{Sq} . \varepsilon=0.3$

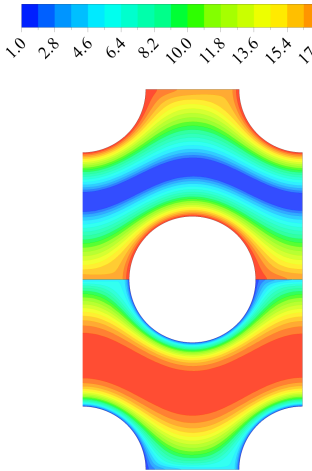

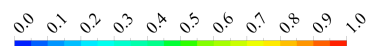

(c) St. $\varepsilon=0.7 ; R e=0.5$
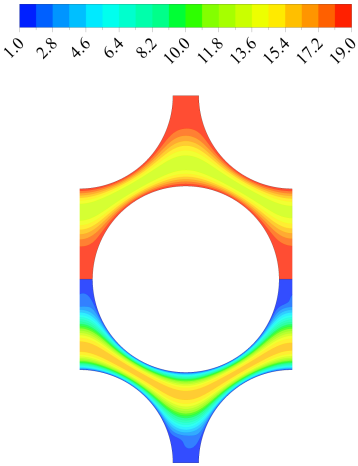

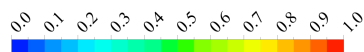

(d) St. $\varepsilon=0.3 ; R e=0.5$

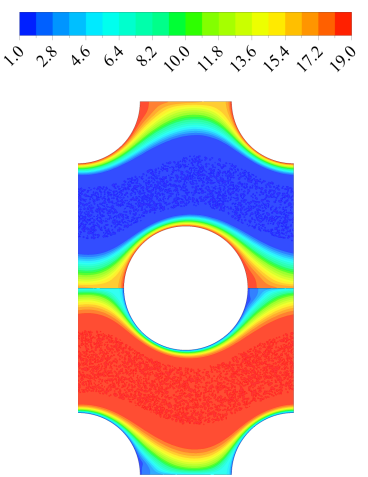

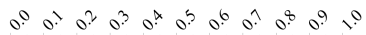

(g) St. $\varepsilon=0.7 ; R e=10$

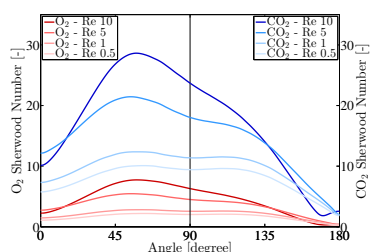

(k) St. $\varepsilon=0.7$

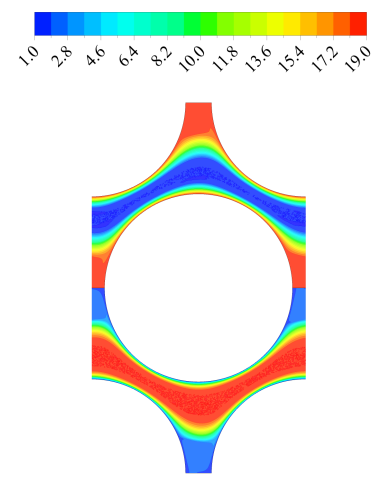

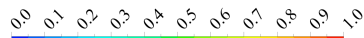

(h) St. $\varepsilon=0.3 ; R e=10$

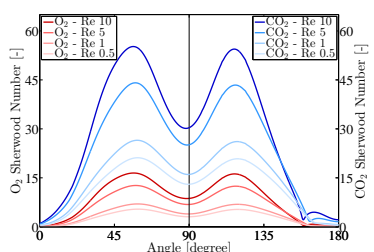

(1) St. $\varepsilon=0.3$

Fig. 5. $\left(\mathrm{a}-\mathrm{h}\right.$ ) Normalised partial pressure of $\mathrm{O}_{2}$ (top half) and $\mathrm{CO}_{2}$ (bottom half) of porosity 0.7 and 0.3 for transverse flow past square (Sq) and staggered (St) configurations at $R e=0.5$ and $R e=10$. (i - I) Local $S h_{\mathrm{O}_{2}}$ and $S h_{\mathrm{CO}_{2}}$ along one fibre of porosity 0.7 and 0.3 at $R e=0.5,1,5$ and 10 transverse flow past square $(\mathrm{Sq})$ and staggered (St) configurations.

identifying the correlation between $S h$ and $\varepsilon$, something that is not explored in the literature a lot. Therefore, parameters $a$ and $b$ (from relation $S h=a R e^{b} S c^{0.33}$ ) are plotted against $\varepsilon$ and presented in a bi-logarithmic format as illustrated for $\mathrm{O}_{2}$ in Figure 6(c) and for $\mathrm{CO}_{2}$ in Figure 6(d). From the curve fits, the functions $a(\varepsilon)$ and $b(\varepsilon)$ can once more be described through power laws with an $\mathrm{R}^{2}$ of 0.99 and above. It should be noted that the relations for parameter $a$ in square and staggered configurations are observed to be different. However, for a given gas $\left(\mathrm{O}_{2}\right.$ or $\left.\mathrm{CO}_{2}\right)$ the relations found for parameter $b$ are interestingly very similar between the different fibre configurations. The generalised mass transfer correlations for square and staggered fibre configurations are tabulated in Table 7 for both $\mathrm{O}_{2}$ and $\mathrm{CO}_{2}$ and are expressed as power law functions of $\varepsilon$. 


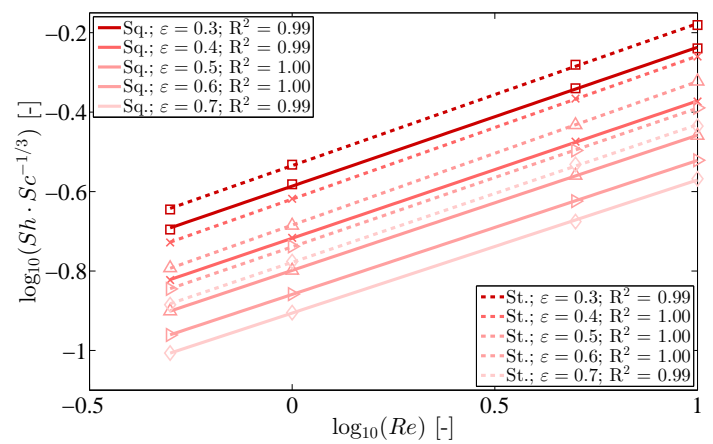

(a) Transv. flow $\mathrm{O}_{2}$ mass transfer correlation

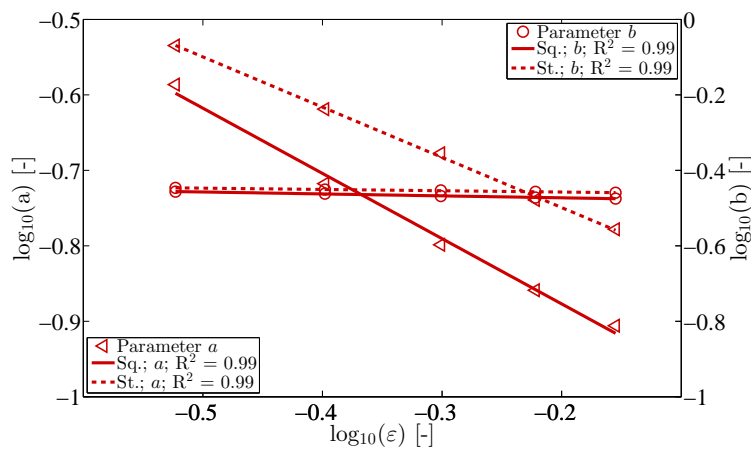

(c) Transv. flow $\mathrm{O}_{2}$ mass transfer parameters

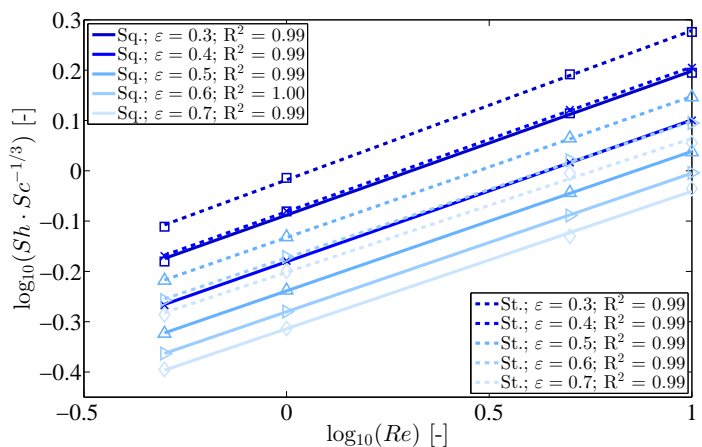

(b) Transv. flow $\mathrm{CO}_{2}$ mass transfer correlation

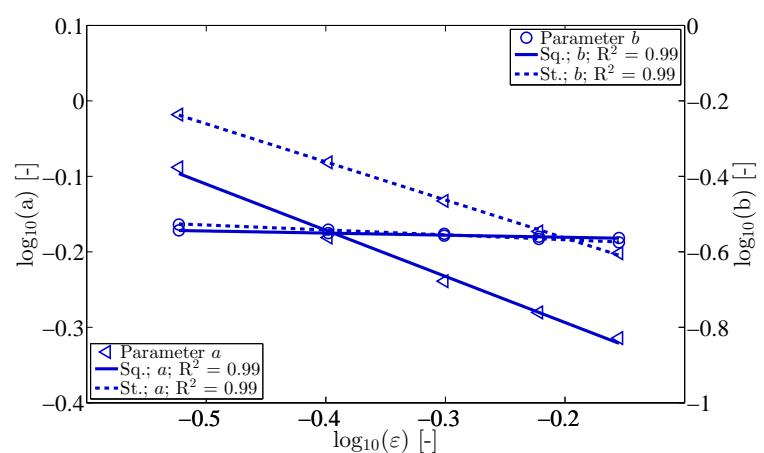

(d) Transv. flow $\mathrm{CO}_{2}$ mass transfer parameters

Fig. 6. Mass transfer correlations comparison of (a) $\mathrm{O}_{2}$ and (b) $\mathrm{CO}_{2}$ for transverse flow at porosities $0.7(\diamond), 0.6(\triangleright), 0.5(\triangle), 0.4(\times)$ and $0.3(\square)$. Mass transfer parameters $a(\triangleleft)$ and $b(\bigcirc)$ fittings of (c) $\mathrm{O}_{2}$ and (d) $\mathrm{CO}_{2}$ for transverse flow. Solid line represents square arrangement and dashed line represents staggered arrangements. Data are fitted via least squares and expressed in lines with the mass transfer and $R^{2}$ correlations. The $R^{2}$ correlations represent the actual fittings without the $\log _{10}$ terms.

\begin{tabular}{c|cc|cc}
\hline \hline$\varepsilon$ & Square $-\mathrm{O}_{2}$ & Square $-\mathrm{CO}_{2}$ & Staggered $-\mathrm{O}_{2}$ & Staggered $-\mathrm{CO}_{2}$ \\
\hline & & & & \\
0.3 & $S h=0.2592 R e^{0.3496} S c^{0.33}$ & $S h=0.8164 R e^{0.2863} S c^{0.33}$ & $S h=0.2920 R e^{0.3577} S c^{0.33}$ & $S h=0.9591 R e^{0.2968} S c^{0.33}$ \\
0.4 & $S h=0.1916 R e^{0.3458} S c^{0.33}$ & $S h=0.6594 R e^{0.2816} S c^{0.33}$ & $S h=0.2405 R e^{0.3600} S c^{0.33}$ & $S h=0.8291 R e^{0.2876} S c^{0.33}$ \\
0.5 & $S h=0.1590 R e^{0.3404} S c^{0.33}$ & $S h=0.5770 R e^{0.2773} S c^{0.33}$ & $S h=0.2068 R e^{0.3614} S c^{0.33}$ & $S h=0.7368 R e^{0.2803} S c^{0.33}$ \\
0.6 & $S h=0.1384 R e^{0.3373} S c^{0.33}$ & $S h=0.5245 R e^{0.2754} S c^{0.33}$ & $S h=0.1823 R e^{0.3492} S c^{0.33}$ & $S h=0.6714 R e^{0.2716} S c^{0.33}$ \\
0.7 & $S h=0.1242 R e^{0.3351} S c^{0.33}$ & $S h=0.4852 R e^{0.2733} S c^{0.33}$ & $S h=0.1667 R e^{0.3466} S c^{0.33}$ & $S h=0.6283 R e^{0.2661} S c^{0.33}$ \\
& & & & \\
\hline \hline
\end{tabular}

Table 6. Transverse flow mass transfer correlation of $\mathrm{O}_{2}$ and $\mathrm{CO}_{2}$ at various $\varepsilon$ in square and staggered configurations.

\begin{tabular}{l|cc}
\hline \hline Gas type & Square & Staggered \\
\hline & & \\
$\mathrm{O}_{2}$ & $S h=0.0892 \varepsilon^{-0.864} R e^{0.3288 \varepsilon^{-0.051}} S c^{0.33}$ & $S h=0.1311 \varepsilon^{-0.666} R e^{0.3433 \varepsilon^{-0.034}} S c^{0.33}$ \\
$\mathrm{CO}_{2}$ & $S h=0.3838 \varepsilon^{-0.611} R e^{0.2676 \varepsilon^{-0.055}} S c^{0.33}$ & $S h=0.5216 \varepsilon^{-0.505} R e^{0.2547 \varepsilon^{-0.130}} S c^{0.33}$ \\
& & \\
\hline \hline
\end{tabular}

Table 7. Findings of $\mathrm{O}_{2}$ and $\mathrm{CO}_{2}$ mass transfer correlation for transverse flow past square and staggered fibre arrangements.

\subsubsection{Parallel Flow Analysis}

Flow: The cases where the flow is perfectly parallel to the fibres are more straightforward to analyse compared to the transverse flow. Figures 7(a) to 7(d) illustrate the normalised velocity magnitude (normalised by its respective maximum velocity) 


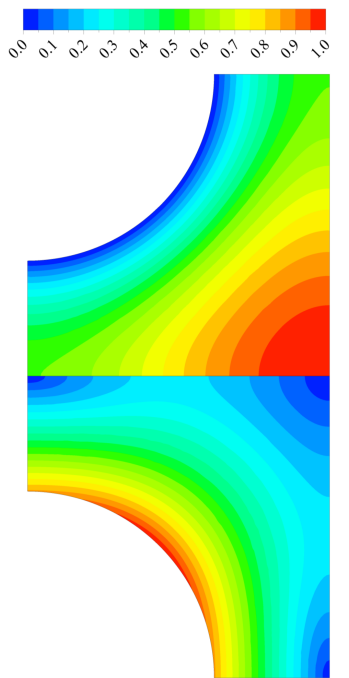

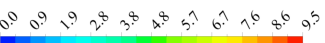

(a) $\mathrm{Sq} ; \varepsilon=0.7 ; \operatorname{Re}=10$

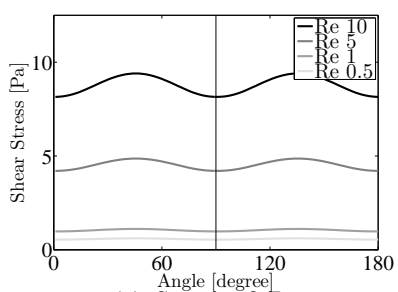

(e) $\mathrm{Sq} ; \varepsilon=0.7$

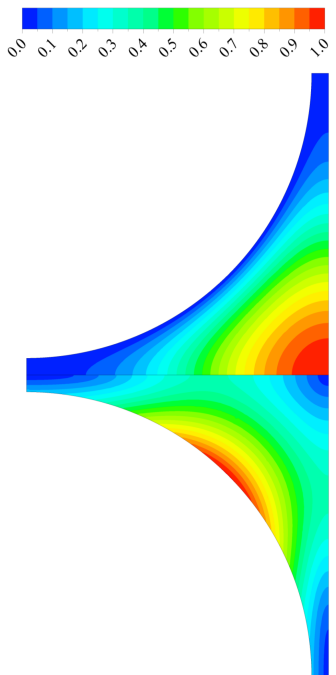

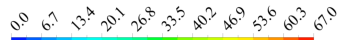

(b) $\mathrm{Sq} ; \varepsilon=0.3 ; \operatorname{Re}=10$

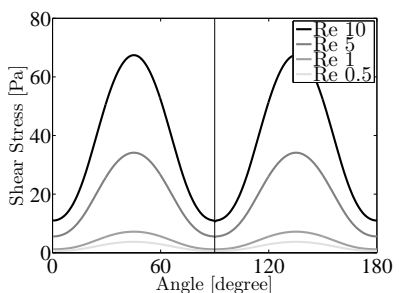

(f) $\mathrm{Sq} ; \varepsilon=0.3$

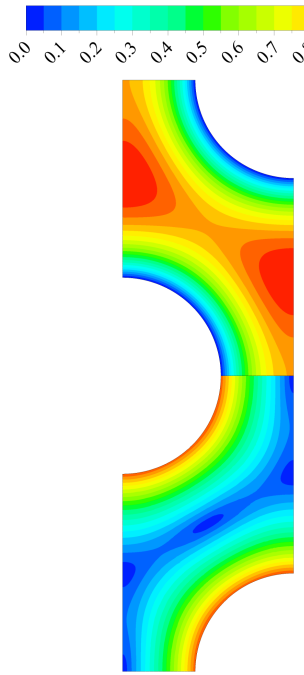

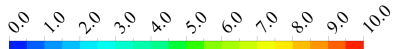

(c) St; $\varepsilon=0.7 ; \operatorname{Re}=10$

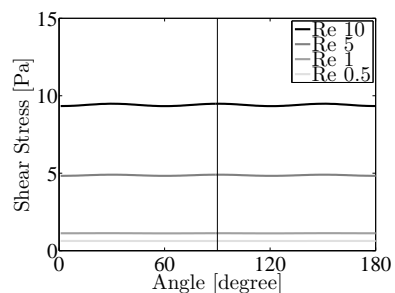

(g) $\mathrm{St} ; \varepsilon=0.7$

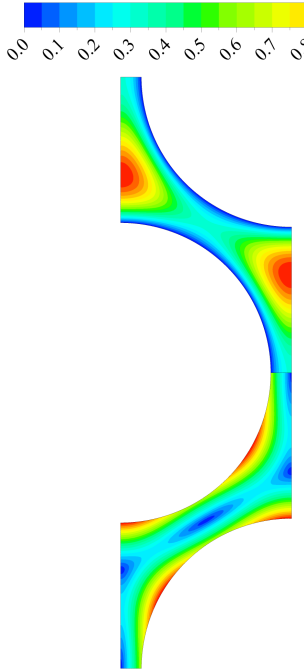

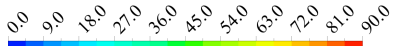

(d) St; $\varepsilon=0.3 ; R e=10$

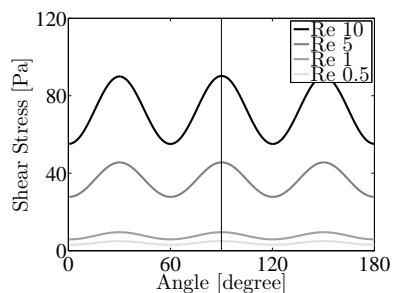

(h) $\mathrm{St} ; \varepsilon=0.3$

Fig. 7. $(a-d)$ Normalised velocity magnitude with streamlines superimposed (top half) and shear stress contours (bottom half) of porosity 0.7 and 0.3 for parallel flow past square (Sq) and staggered (St) configurations at $R e=10$. Contour legend in the top and bottom depicts the normalised velocity and shear stress respectively. $(\mathrm{e}-\mathrm{h})$ Wall shear stress along one fibre of porosity 0.7 and 0.3 at $\operatorname{Re}=0.5,1,5$ and 10 transverse flow past square (Sq) and staggered (St) configurations.

and shear stress contours for both configurations at $R e=10$. As expected, it is evident from the velocity distributions for both configurations that high velocity regions occur in areas of low resistance. Figures 7(e) to 7(h) depict that the highest shear stress region is observed at the wall situated closest to location where the bulk of the fluid flows. Similar to the transverse flow, fluid in contact with the fibre region is large at $\varepsilon=0.7$ which results in a wide profile of shear stress, illustrated in Figure 7(e) at $R e=0.5$. As $\varepsilon$ decreases, the shear stress profile appears to be thinner with a higher magnitude which is caused by the decreasing regions of fluid contacting the fibre (Figure 7(f) at $R e=0.5$ ) which resulted in a large difference in their respective shear stress along the fibre. Similar trends can be observed for staggered configurations with three peaks caused by the nature of the geometry configuration, depicted in Figures 7(g) and 7(h). The gaps in between fibres are slightly larger at $\varepsilon=0.3$ than the square configuration resulting in a higher shear stress magnitude. The peak appears at $45^{\circ}$ and $135^{\circ}$ for square arrangements and at $30^{\circ}, 90^{\circ}$ and $150^{\circ}$ for staggered configurations. As Re increases, the magnitude is observed to be higher with a clearer profile for all porosities.

Oxygenation: Figures $8\left(\right.$ a) to $8(\mathrm{~h})$ depict partial pressures of $\mathrm{O}_{2}$ and $\mathrm{CO}_{2}$, normalised by their respective initial partial pressures, for flow parallel to the fibre in square and staggered configurations for porosities of 0.7 and 0.3 at $R e$ of 0.5 and 
10. Contours are displayed separately at the top and bottom halves of the domain for $\mathrm{O}_{2}$ and $\mathrm{CO}_{2}$. Similar trends can be found for parallel as observed in transverse flows, i.e. for a given flow gas transfer improves for decreasing $\varepsilon$.

Figures 8(i) to 8(1) illustrate the circumferential $S h_{\mathrm{L}, \mathrm{O}_{2}}$ and $S h_{\mathrm{L}, \mathrm{CO}_{2}}$ profiles of one fibre. It can be seen that the $S h_{\mathrm{L}, \mathrm{O}_{2}}$ and $S h_{\mathrm{L}, \mathrm{CO}_{2}}$ are approximately constant at $\varepsilon=0.7$ (Figure 8(i)) and tend to be thinner as $\varepsilon$ decreases (Figure 8(j)). The peak positions of $S h_{\mathrm{L}, \mathrm{O}_{2}}$ and $S h_{\mathrm{L}, \mathrm{CO}_{2}}$ are found to be located at $45^{\circ}$ and $135^{\circ}$. The upstream and downstream $S h_{\mathrm{L}}$ reduce to zero as $\varepsilon$ decreases, which shows the possibility of a stagnation region with limited gas transfer occurs as illustrated in Figure 8(j). For staggered configurations, the $S h_{\mathrm{L}}$ profiles appeared to be constant for both porosity 0.7 and 0.3 , illustrated in Figures $8(\mathrm{k})$ and $8(\mathrm{l})$. Peak values are visible for $\varepsilon=0.3$ (Figure $8(1)$ ) and is found to be located at $30^{\circ}, 90^{\circ}$ and $150^{\circ}$. The upstream and downstream $S h_{\mathrm{L}}$ does not reduce to zero due to their geometrical nature.

As $R e$ increases, the partial pressure boundary layers for $\mathrm{O}_{2}$ and $\mathrm{CO}_{2}$ in both arrangements (Figures 8(e) to 8(h)) appeared to be much thinner which implies that the gas transfer at the fibre surface is improved. The trends of circumferential $S h_{\mathrm{L}, \mathrm{O}_{2}}$ and $S h_{\mathrm{L}, \mathrm{CO}_{2}}$ profiles are similar with clearer peak magnitudes.

Mass transfer correlation: Figures 9(a) and 9(b) depict the least squares fitted mass transfer correlations of $\mathrm{O}_{2}$ and $\mathrm{CO}_{2}$ at various $\varepsilon$ for both square and staggered configurations. Similar to the fittings of mass transfer correlations for the transverse flow, the correlations for parallel flow are fitted as linear correlations, in logarithmic form, with an $\mathrm{R}^{2}$ correlation of 0.99 and above. Interestingly, it is observed that porosity 0.3 and 0.4 of the square configuration appear to be steeper slopes for $\mathrm{O}_{2}$ and $\mathrm{CO}_{2}$. This is due to the flow path on the particular small fibre spacing, which can lead to stagnant areas especially at low $R e$, reducing the gas transfer area. This phenomena improves when $R e$ is increased. The fitted power laws mass transfer correlations are tabulated in Table 8 for square and staggered configurations. Again a generalisation can be performed by identifying the $\varepsilon$ dependency of parameters a and $\mathrm{b}$. These correlations are presented in a bi-logarithmic format as illustrated in Figures 9(c) for $\mathrm{O}_{2}$ and $9(\mathrm{~d})$ for $\mathrm{CO}_{2}$. According to the results, the functions $a(\varepsilon)$ and $b(\varepsilon)$ can be described through power laws with an $\mathrm{R}^{2} 0.96$ and above. The generalised mass transfer correlations for square and staggered fibre arrangements are tabulated in Table 9 for both $\mathrm{O}_{2}$ and $\mathrm{CO}_{2}$ and are expressed as power law functions of $\varepsilon$.

\begin{tabular}{l|cc|cc}
\hline \hline$\varepsilon$ & Square $-\mathrm{O}_{2}$ & Square $-\mathrm{CO}_{2}$ & Staggered $-\mathrm{O}_{2}$ & Staggered $-\mathrm{CO}_{2}$ \\
\hline & & & & \\
0.3 & $S h=0.0257 R e^{0.6897} S c^{0.33}$ & $S h=0.4534 R e^{0.4599} S c^{0.33}$ & $S h=0.1393 R e^{0.3297} S c^{0.33}$ & $S h=1.3149 R e^{0.2470} S c^{0.33}$ \\
0.4 & $S h=0.0682 R e^{0.4207} S c^{0.33}$ & $S h=0.7499 R e^{0.2911} S c^{0.33}$ & $S h=0.1179 R e^{0.3159} S c^{0.33}$ & $S h=1.1770 R e^{0.2359} S c^{0.33}$ \\
0.5 & $S h=0.0832 R e^{0.2825} S c^{0.33}$ & $S h=0.8738 R e^{0.2204} S c^{0.33}$ & $S h=0.1024 R e^{0.2883} S c^{0.33}$ & $S h=1.0239 R e^{0.2319} S c^{0.33}$ \\
0.6 & $S h=0.0772 R e^{0.2675} S c^{0.33}$ & $S h=0.8440 R e^{0.2241} S c^{0.33}$ & $S h=0.0900 R e^{0.2738} S c^{0.33}$ & $S h=0.9046 R e^{0.2229} S c^{0.33}$ \\
0.7 & $S h=0.0649 R e^{0.2576} S c^{0.33}$ & $S h=0.7495 R e^{0.2265} S c^{0.33}$ & $S h=0.0712 R e^{0.2612} S c^{0.33}$ & $S h=0.8103 R e^{0.2189} S c^{0.33}$ \\
& & & & \\
\hline \hline
\end{tabular}

Table 8. Parallel flow mass transfer correlation of $\mathrm{O}_{2}$ and $\mathrm{CO}_{2}$ at various $\varepsilon$ in square and staggered configurations. 


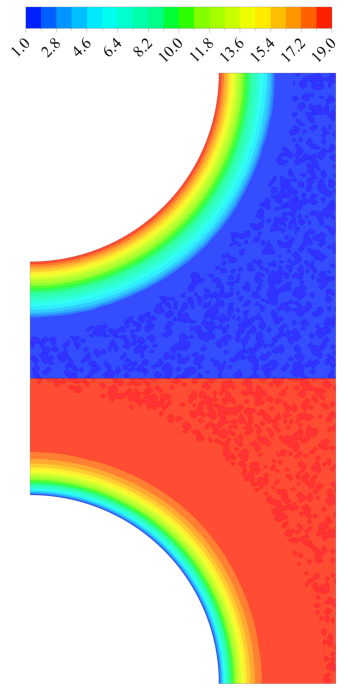

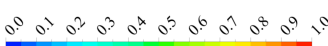

(a) Sq. $\varepsilon=0.7 ; \operatorname{Re}=0.5$

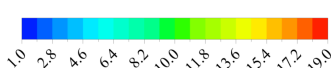

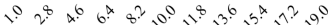

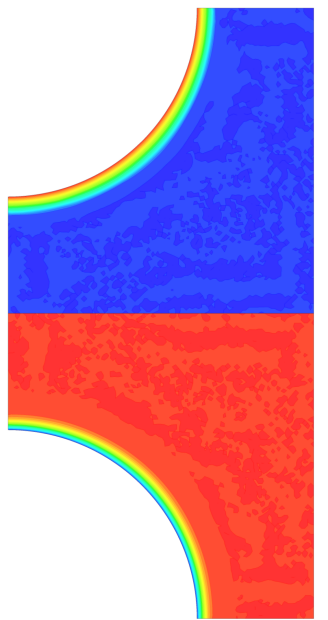

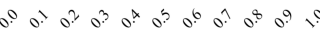

(e) Sq. $\varepsilon=0.7 ; \operatorname{Re}=10$

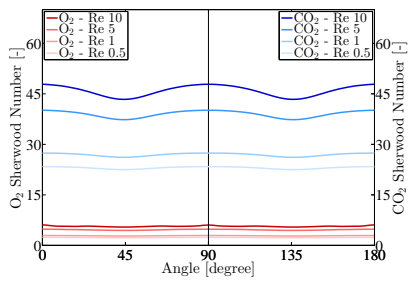

(i) Sq. $\varepsilon=0.7$

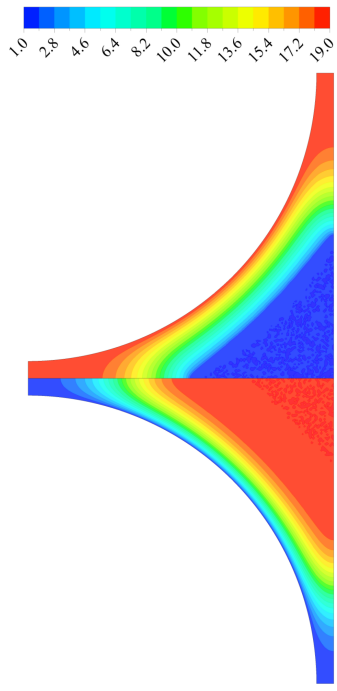

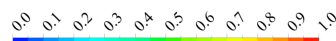

(b) Sq. $\varepsilon=0.3 ; \operatorname{Re}=0.5$

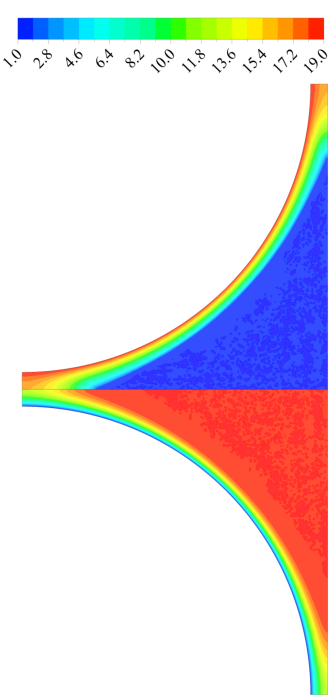

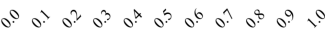

(f) Sq. $\varepsilon=0.3 ; \operatorname{Re}=10$

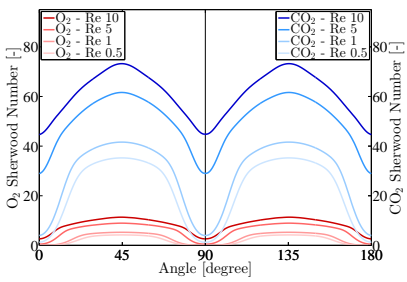

(j) Sq. $\varepsilon=0.3$
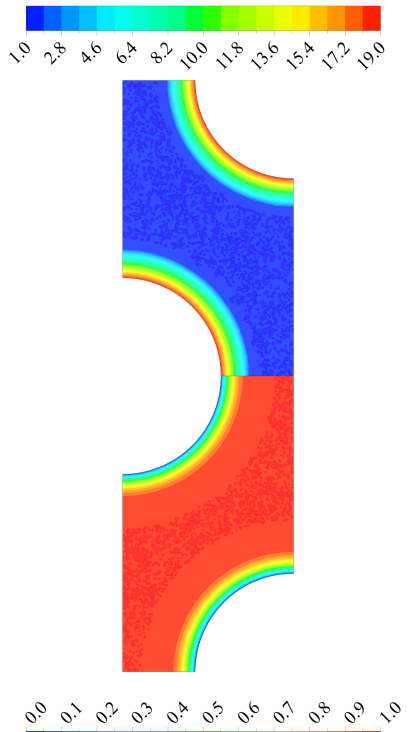

(c) St. $\varepsilon=0.7 ; \operatorname{Re}=0.5$
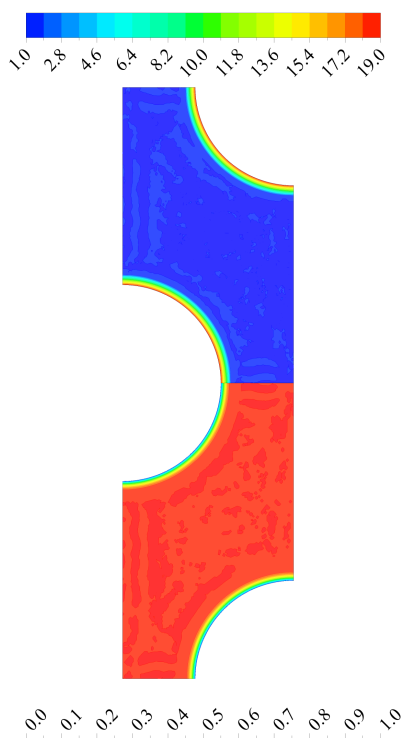

(g) St. $\varepsilon=0.7 ; R e=10$

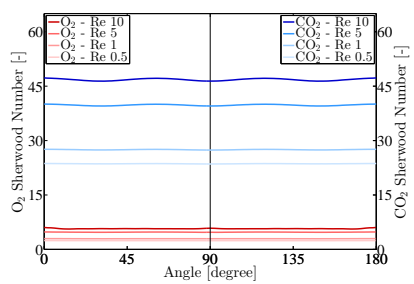

(k) St. $\varepsilon=0.7$

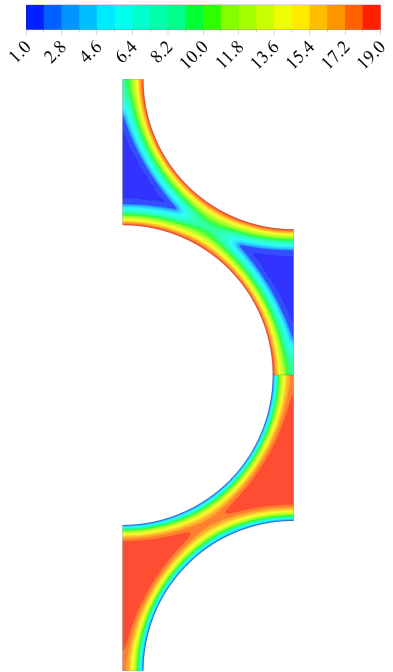

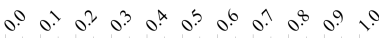

(d) St. $\varepsilon=0.3 ; R e=0.5$

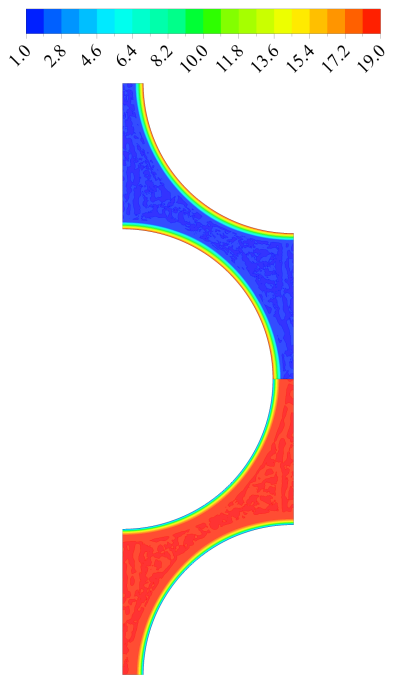

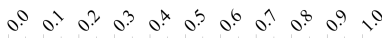

(h) St. $\varepsilon=0.3 ; \operatorname{Re}=10$

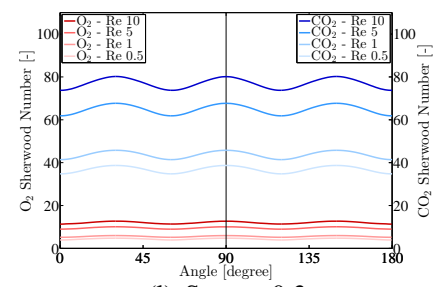

(1) St. $\varepsilon=0.3$

Fig. 8. (a $-\mathrm{h})$ Normalised partial pressure of $\mathrm{O}_{2}$ (top half) and $\mathrm{CO}_{2}$ (bottom half) of porosity 0.7 and 0.3 for parallel flow past square (Sq) and staggered (St) configurations at $R e=0.5$ and $R e=10$. (i - I) Local $S h_{\mathrm{O}_{2}}$ and $S h_{\mathrm{CO}_{2}}$ along one fibre of porosity 0.7 and 0.3 at $R e=0.5$, 1,5 and 10 parallel flow past square $(\mathrm{Sq})$ and staggered $(\mathrm{St})$ configurations.

\section{Discussions}

The development of mass transfer correlations have been carried out in the previous section for transverse flow (Section 3.3.1) and parallel flow (Section 3.3.2). The mass transfer correlations are well-defined functions of the $\varepsilon$ and are found to 


\begin{tabular}{l|cc}
\hline \hline Gas type & Square & Staggered \\
\hline & & \\
$\mathrm{O}_{2}$ & $S h=0.0180 \varepsilon^{-8.7133 \log (\varepsilon)-4.8654} R e^{0.3050 \varepsilon^{3.2197 \log (\varepsilon)+0.9927} S c^{0.33}}$ & $S h=0.0584 \varepsilon^{-0.751} R e^{0.237 \varepsilon^{-0.286}} S c^{0.33}$ \\
$\mathrm{CO}_{2}$ & $S h=0.3814 \varepsilon^{-4.7273 \log (\varepsilon)-2.6162} R e^{0.3310 \varepsilon^{3.6219 \log (\varepsilon)+1.6150} S c^{0.33}}$ & $S h=0.6735 \varepsilon^{-0.578} R e^{0.2082 \varepsilon^{-0.142}} S c^{0.33}$ \\
& & \\
\hline \hline
\end{tabular}

Table 9. Findings of $\mathrm{O}_{2}$ and $\mathrm{CO}_{2}$ mass transfer correlation for parallel flow past square and staggered arrangements.

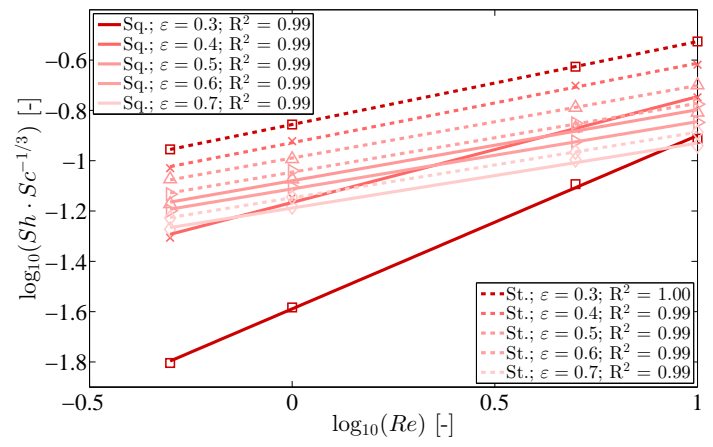

(a) Parallel flow $\mathrm{O}_{2}$ mass transfer correlation

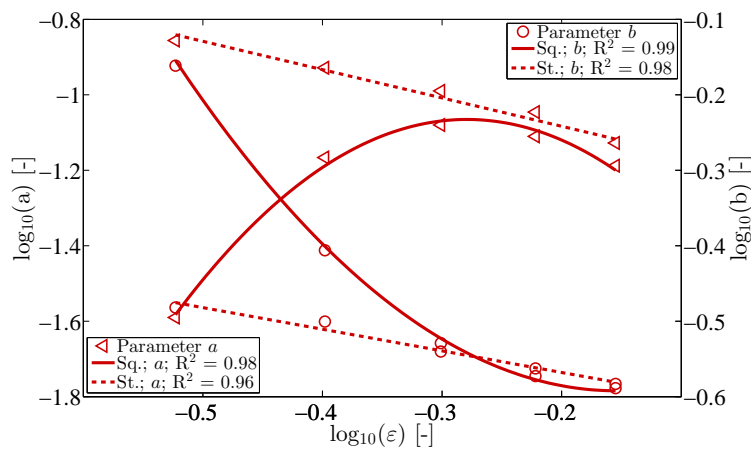

(c) Parallel flow $\mathrm{O}_{2}$ mass transfer parameters

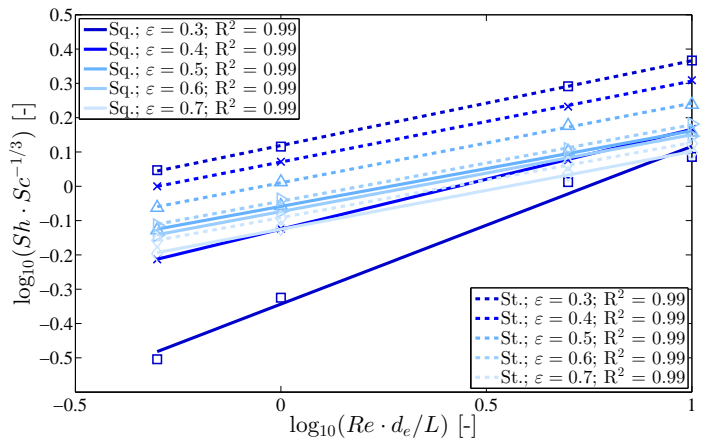

(b) Parallel flow $\mathrm{CO}_{2}$ mass transfer correlation

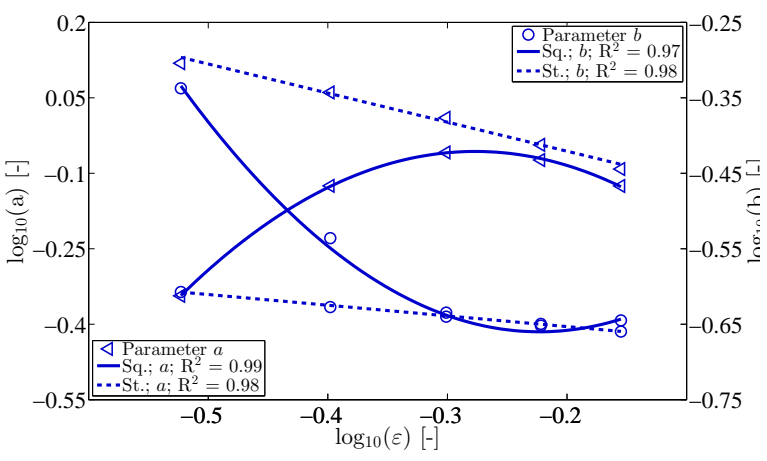

(d) Parallel flow $\mathrm{CO}_{2}$ mass transfer parameters

Fig. 9. Mass transfer correlations comparison of (a) $\mathrm{O}_{2}$ and (b) $\mathrm{CO}_{2}$ for parallel flow at porosities $0.7(\diamond), 0.6(\triangleright), 0.5(\triangle), 0.4(\times)$ and $0.3(\square)$. Mass transfer parameters $a(\triangleleft)$ and $b(\bigcirc)$ fittings of (c) $\mathrm{O}_{2}$ and (d) $\mathrm{CO}_{2}$ for parallel flow. Solid line represents square arrangement and dashed line represents staggered arrangements. Data are fitted via least squares and expressed in lines with the mass transfer and $\mathrm{R}^{2}$ correlations. The $\mathrm{R}^{2}$ correlations represent the actual fittings without the $\log _{10}$ terms.

be expressed as power law functions for their respective parameters $a$ and $b$. In this section, an example is given on how to use the correlations found in previous sections for the design of a blood oxygenator. Without performing any CFD, the gas transfer can be determined or compared for: (1) different flow directions, (2) fibre configurations and (3) porosities.

A blood oxygenator size of width $(w=6.5 \mathrm{~cm})$, height $(h=6.5 \mathrm{~cm})$ and length $(l=15 \mathrm{~cm})$, adopted from the work of Dierickx et al. [13], is used in this comparison. The aforementioned dimensions are based on the same cross-sectional area of the device with an average fibre length. Fibres are arranged in a manner that the flow is either perpendicular or parallel across the whole length of the device. This investigation is conducted at porosities ranging from 0.3 to 0.7 . Furthermore, additional checks are carried out to ensure that cut-off points for transverse flow adopted in Section 3.2 remains valid for the defined porosity range. This can be approximated by dividing the width/height of the device by the spacing of each porosity and fibre configuration. We found that the maximum error relative to the cut-off point (125 fibres across one direction) 
for both configurations is $5.23 \%$. Therefore, the range of porosity (0.3 to 0.7 ) for this comparison is acceptable. Diameter of fibres are kept the same $(380 \mu \mathrm{m})$ and are arranged as square or staggered configurations. A flow rate $(Q)$ of $5 \mathrm{~L} / \mathrm{min}$ is prescribed at the inlet. An average velocity, $\bar{u}$, is computed from the flow rate which will subsequently be used for $R e$ calculations. The prescribed flow rate is divided by the frontal fluid area, which is the net fluid area of the surface normal to the bulk flow direction. Reynolds number calculations are based on a blood density of $1,050 \mathrm{~kg} / \mathrm{m}^{3}$, a constant viscosity at high shear rate range of $0.00345 \mathrm{~Pa} \cdot \mathrm{s}$ and the fibre diameter. The Re computed for transverse flow ranges from 4 to 10 for square and staggered fibre arrangements. In contrast, the Re calculated for parallel flow ranges from 2 to 6 for both configurations. Incorporating the correct $a$ and $b$ parameters, the overall mass transfer coefficient $(k)$ can be evaluated using the relation $S h_{i}=k_{i} d_{h} / D_{\text {eff }, i}$ for $\mathrm{O}_{2}$ and $\mathrm{CO}_{2}$. The hydraulic diameter, $d_{h}$, is defined as

$$
d_{h}=\frac{4 V_{\mathrm{B}}}{A_{\mathrm{surf}}} ; \quad V_{\mathrm{B}}=\left\{\begin{array}{ll}
\left(\frac{\pi d^{2}}{S_{\mathrm{P}}^{2}}\right) w \cdot h \cdot l & \text { transverse; } \\
\left(1-\frac{\pi d^{2}}{4 S_{\mathrm{p}}^{2}}\right) w \cdot h \cdot l & \text { parallel; }
\end{array} \quad A_{\text {surf }}= \begin{cases}\left(\frac{\pi d}{S_{\mathrm{p}}^{2}}\right) w \cdot h \cdot l & \text { transverse } \\
\left(\frac{\pi d}{S_{\mathrm{p}}^{2}}\right) w \cdot h \cdot l & \text { parallel }\end{cases}\right.
$$

where $V_{\mathrm{B}}, A_{\text {surf }}$ and $S_{\mathrm{p}}$ denote the blood volume in the packed fibre that is open to the flow, surface area of the fibres that is resisting the flow and spacing between fibre centres, respectively.

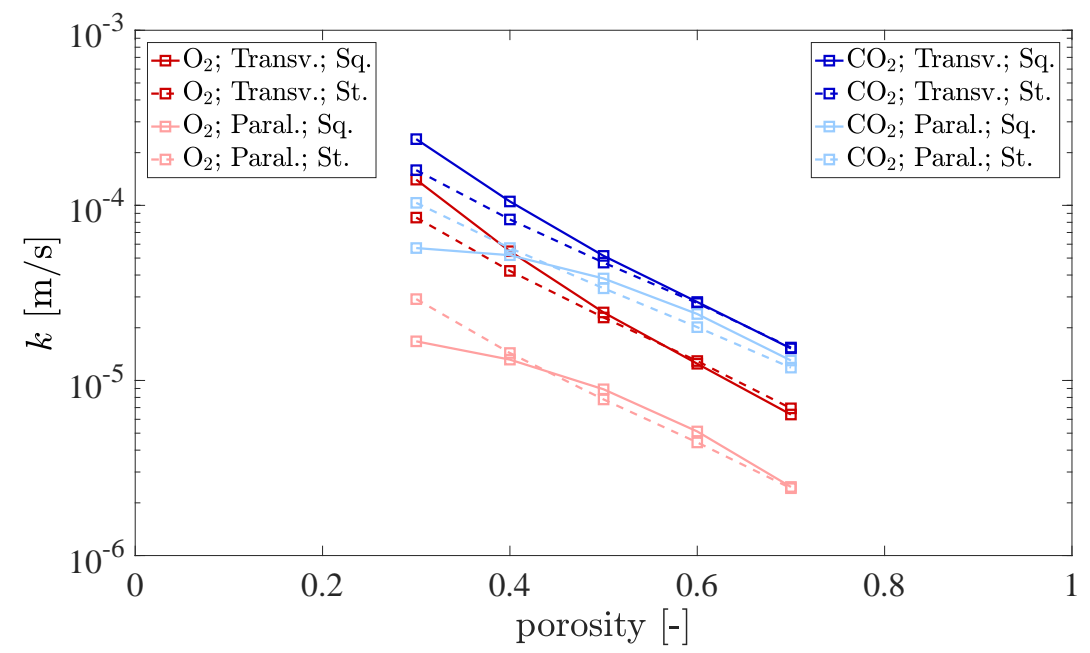

Fig. 10. Mass transfer coefficients comparison between fibre configurations and flow directions at various $\varepsilon$ levels for (a) $\mathrm{O}_{2}$ and (b) $\mathrm{CO}_{2}$. The notation Sq. refers to square and St. refers to staggered fibre configuration. The notation Transv. represents transverse flow and Paral. represents parallel flow.

Figure 10 illustrates the computed $\mathrm{O}_{2}$ and $\mathrm{CO}_{2}$ mass transfer coefficients of different fibre configurations and flow directions at various porosity levels. It can be observed in general that the transverse flow has better gas transfer performance compared to parallel flow. The difference in flow direction appears to be apparent for $\mathrm{O}_{2}$ with difference relative to their respective configuration ranging between $61 \%$ and $88 \%$. In contrast, the difference in $\mathrm{CO}_{2}$ mass transfer coefficient relative to their respective configuration is found to be smaller with the range between $2 \%$ and $50 \%$. Furthermore, the effect of fibre 
configuration dependency is observed to be subtle at $\varepsilon=0.7$ and gradually increases as porosity increases. However, at $\varepsilon=0.3$, it can be seen that the fibre configuration is extremely sensitive towards the oxygenation performance. The square fibre arrangement is observed to have a higher mass transfer coefficient (better gas transfer) from porosity 0.3 to 0.5 when the flow is perpendicular to the fibres. As for parallel flow, this behaviour tends to be contradicting.

It is worth comparing our findings with reported literature. To the authors' best knowledge, there have not been any investigations performed between different flow directions and fibre configurations using numerical simulations. To date, there are only two studies available in the literature, the work of Yang and Cussler [29] and Wickramasinghe et al. [6], who have successfully compared mass transfer of two different flow directions experimentally. Qualitative trends are observed and the findings are consistent with ours, stating that transverse flow has better gas transfer than parallel flow. However, we found difficulty in conducting appropriate comparisons between the magnitudes of $a$ and $b$ parameters with the reported literature tabulated in Tables 1 and 2. This is due to the lack of information in the description of geometrical features (e.g. shape and dimensions), experimental procedures (e.g. experimental fluid and gas type(s) for oxygenation) and operating conditions (e.g. flow rates). More importantly, the experiments for comparisons are mainly conducted on commercial and handmade prototypes using water to establish the mass transfer correlations whereas the current work focuses on the establishment of mass transfer correlations based on perfect fibres in an idealised configuration. It is observed that the experiments conducted are based on well-spaced fibre that are manually folded which may have a tendency of randomness compared to the idealised fibre configurations in our work. Also, end wall effects contain the possibility of redistributing the flow within the device which could affect the gas transfer. Furthermore, the consideration of the velocity term in the $R e$ from these works are unclear and it is highly important as it will affect the Sherwood number and, subsequently, the mass transfer coefficient. For instance, if the velocity is computed based on the inflow, where the area is usually smaller than the area within the device, the $R e$ will increase leading to a shift in mass transfer correlations and subsequently providing an inaccurate mass transfer coefficient. Therefore, it is important to ensure that an appropriate $R e$, based on the fibre bundle is used prior to calculating the mass transfer correlations and mass transfer coefficient.

We believe that this rigorous numerical investigation aimed at establishing mass transfer correlations will enable readers to initially assess and improve gas transfer performance on device prototypes without the need to conduct any necessary CFD simulations or experimental works. Furthermore, mass transfer correlations at present are typically fitted experimentally based on a whole blood oxygenator and it would be difficult to understand the underlying mechanism of oxygenation when one is looking to improve the performance of the device. Therefore, this work employs an approach to numerically investigate the problem based on first principles to really appreciate the ongoing oxygenation phenomenon. A summary of the procedure to provide readers with some guidelines on the methodology for evaluating the performance in a blood oxygenator is laid out as below:

1. Extract information: device dimension, fibre diameter, fibre configuration and flow direction.

2. Calculate device porosity and maximum fibre across one direction (transverse flow) or fibre length (parallel flow).

3. Based on the porosity and flow direction, choose the correct expression for $\overline{S h}-$ refer to Section 3.2. 
4. According to 2, compute representable $S h \equiv \overline{S h}-$ refer to Section 3.2 Eqn. 14.

5. Establish mass transfer correlation $\left(S h=a R e^{b} S c^{0.33}\right)$ and identify parameters $a$ and $b$ - analogous to mass transfer correlation in Sections 3.3.1 and 3.3.2.

6. Compute $\bar{u}, R e$ and $d_{h}$ according to the device operating condition - refer to paragraph 2 of Section 4 .

7. Utilise the mass transfer correlation from 5 to compute $S h$.

8. Compute the overall mass transfer coefficient, $k=S h \cdot D_{\text {eff }} / d_{h}$.

If the fibre calculations are very close to the ones presented in this work, skip Step $3-5$. Step 6 remains essential. The next step is to choose the appropriate $a$ and $b$ parameters from established mass transfer correlation $S h=a(\varepsilon) R e^{b(\varepsilon)} S c^{0.33}$ in Table 7 or Table 9 and finally repeating Step 8 to evaluate the mass transfer coefficient.

\section{Conclusions}

Generalised $\mathrm{O}_{2}$ and $\mathrm{CO}_{2}$ mass transfer correlations for non-Newtonian transverse and parallel blood flow in uniformly arranged fibre bundles have been established numerically in order to provide an overall mass transfer description at oxygenator scale. Transverse and parallel blood flow and $\mathrm{O}_{2}$ and $\mathrm{CO}_{2}$ transport behaviour in an array of fibres being arranged in a square and staggered manner is being studied. The systematic investigation covers a range of $\varepsilon$ levels which are found in typical commercial oxygenators and leads to correlations of the form $S h=a(\varepsilon) R e^{b(\varepsilon)} S c^{0.33}$.

An important investigation on the development of concentration across the domain is conducted and a set of power law expressions for $\overline{S h}$ is established which covers the range from a deoxygenated blood condition to a fully oxygenated blood state. In order to obtain a representable $\overline{S h}$ for the whole domain, a cut-off point based on the number of fibres or length is required to avoid under- or over-estimation. A typical commercial oxygenator feature is used to further demonstrate the methodology. The final generalised mass transfer correlations consist of eight geometrical, flow and gas type dependent expressions. They are found to be power law functions expressed as a function of $\varepsilon$ as compared to constants reported in literature. The inclusion of $\varepsilon$ dependency is proven to be essential, as presented in the numerical results, where the local Sherwood number varies significantly. The use of these mass transfer correlations are performed to determine the performance of gas transfer in a typical oxygenator size with a standard operating condition at various porosity level, fibre configuration and flow direction. Overall, the gas transfer for transverse flow is found to be better than parallel flow and is consistent with reported literature. Furthermore, the effect of fibre configuration is pronounced when porosity is lower than 0.5 . The square configuration in the transverse flow performs better with aforementioned porosity range. However, an opposite effect is observed for parallel flow.

Despite the lack of reported information in experiments for comparison purposes, this set of mass transfer correlations based on rigorous numerical investigation will enable users to initially assess their prototype gas performance without the need to conduct any necessary CFD simulations and experiments. Future works include the consideration of end wall effects on the mass transfer correlations as they could redistribute the flow magnitude. Furthermore, this work could also be further extended by investigating the effect of pulsatility on the oxygenation and mass transfer correlations as it promote gas 
mixing [28], which could potentially further enhance the gas transfer efficiency.

\section{Appendix}

6.1 Fitted parameters of power law functions for transverse flow: $\overline{S h}_{\perp, i}=\phi N_{\mathrm{f}}^{-\xi}$

\begin{tabular}{c|c|rccc|rrrr}
\hline \hline & & \multicolumn{7}{|c|}{ Square } & \multicolumn{4}{c}{ Staggered } \\
\hline$\varepsilon$ & $R e$ & $\phi-\mathrm{O}_{2}$ & $\xi-\mathrm{O}_{2}$ & $\phi-\mathrm{CO}_{2}$ & $\xi-\mathrm{CO}_{2}$ & $\phi-\mathrm{O}_{2}$ & $\xi-\mathrm{O}_{2}$ & $\phi-\mathrm{CO}_{2}$ & $\xi-\mathrm{CO}_{2}$ \\
\hline 0.7 & 0.5 & 3.462 & -0.323 & 14.280 & -0.266 & 5.396 & -0.370 & 21.140 & -0.307 \\
0.7 & 1.0 & 4.355 & -0.322 & 17.260 & -0.267 & 6.757 & -0.363 & 25.770 & -0.306 \\
0.7 & 5.0 & 7.629 & -0.331 & 27.150 & -0.275 & 11.520 & -0.354 & 41.050 & -0.311 \\
0.7 & 10.0 & 10.270 & -0.345 & 34.970 & -0.288 & 12.980 & -0.325 & 49.170 & -0.323 \\
\hline 0.6 & 0.5 & 3.984 & -0.333 & 15.900 & -0.276 & 6.168 & -0.382 & 23.600 & -0.319 \\
0.6 & 1.0 & 5.081 & -0.335 & 19.340 & -0.277 & 7.697 & -0.375 & 28.730 & -0.319 \\
0.6 & 5.0 & 9.040 & -0.346 & 31.210 & -0.288 & 13.060 & -0.367 & 45.870 & -0.326 \\
0.6 & 10.0 & 11.980 & -0.358 & 39.680 & -0.301 & 15.250 & -0.341 & 56.100 & -0.335 \\
\hline 0.5 & 0.5 & 4.728 & -0.345 & 18.020 & -0.285 & 7.246 & -0.393 & 26.890 & -0.331 \\
0.5 & 1.0 & 6.009 & -0.344 & 22.040 & -0.287 & 9.113 & -0.388 & 32.790 & -0.330 \\
0.5 & 5.0 & 10.810 & -0.354 & 35.930 & -0.299 & 14.730 & -0.359 & 52.940 & -0.338 \\
0.5 & 10.0 & 14.200 & -0.366 & 45.360 & -0.312 & 18.610 & -0.354 & 66.120 & -0.345 \\
\hline 0.4 & 0.5 & 5.896 & -0.356 & 21.310 & -0.296 & 8.751 & -0.405 & 31.420 & -0.343 \\
0.4 & 1.0 & 7.545 & -0.355 & 26.190 & -0.297 & 11.000 & -0.398 & 38.470 & -0.342 \\
0.4 & 5.0 & 13.730 & -0.367 & 43.470 & -0.313 & 18.570 & -0.382 & 62.940 & -0.350 \\
0.4 & 10.0 & 17.950 & -0.377 & 54.700 & -0.327 & 23.580 & -0.380 & 78.450 & -0.357 \\
\hline 0.3 & 0.5 & 8.465 & -0.374 & 28.240 & -0.320 & 11.070 & -0.417 & 38.270 & -0.361 \\
0.3 & 1.0 & 10.870 & -0.370 & 34.720 & -0.313 & 13.920 & -0.409 & 46.690 & -0.354 \\
0.3 & 5.0 & 19.870 & -0.384 & 57.260 & -0.328 & 23.710 & -0.395 & 76.030 & -0.358 \\
0.3 & 10.0 & 25.590 & -0.390 & 70.530 & -0.334 & 31.050 & -0.407 & 93.670 & -0.362 \\
\hline \hline
\end{tabular}

Table 10. Transverse flow fitted parameters of power law functions for square (left) and staggered (right) configurations.

6.2 Fitted parameters of power law functions for parallel flow: $\overline{S h}_{\|, i}=\phi z^{-\xi}$

\begin{tabular}{c|c|cccc|cccc}
\hline \hline & & \multicolumn{5}{|c|}{ Square } & \multicolumn{4}{c}{ Staggered } \\
\hline$\varepsilon$ & $R e$ & $\phi-\mathrm{O}_{2}$ & $\xi-\mathrm{O}_{2}$ & $\phi-\mathrm{CO}_{2}$ & $\xi-\mathrm{CO}_{2}$ & $\phi-\mathrm{O}_{2}$ & $\xi-\mathrm{O}_{2}$ & $\phi-\mathrm{CO}_{2}$ & $\xi-\mathrm{CO}_{2}$ \\
\hline 0.7 & 0.5 & 0.228 & -0.328 & 4.514 & -0.230 & 0.264 & -0.312 & 5.063 & -0.218 \\
0.7 & 1.0 & 0.279 & -0.328 & 5.313 & -0.229 & 0.318 & -0.314 & 5.891 & -0.218 \\
0.7 & 5.0 & 0.413 & -0.340 & 7.588 & -0.232 & 0.461 & -0.328 & 8.430 & -0.220 \\
0.7 & 10.0 & 0.447 & -0.358 & 8.622 & -0.237 & 0.483 & -0.349 & 9.478 & -0.226 \\
\hline 0.6 & 0.5 & 0.277 & -0.319 & 5.102 & -0.227 & 0.345 & -0.296 & 6.018 & -0.209 \\
0.6 & 1.0 & 0.344 & -0.318 & 6.008 & -0.226 & 0.429 & -0.295 & 7.133 & -0.208 \\
0.6 & 5.0 & 0.522 & -0.329 & 8.637 & -0.229 & 0.639 & -0.307 & 10.440 & -0.208 \\
0.6 & 10.0 & 0.576 & -0.344 & 9.829 & -0.234 & 0.682 & -0.325 & 11.960 & -0.212 \\
\hline 0.5 & 0.5 & 0.284 & -0.330 & 5.121 & -0.237 & 0.412 & -0.292 & 6.732 & -0.210 \\
0.5 & 1.0 & 0.359 & -0.328 & 6.028 & -0.237 & 0.516 & -0.291 & 7.961 & -0.209 \\
0.5 & 5.0 & 0.558 & -0.337 & 8.534 & -0.243 & 0.792 & -0.300 & 11.710 & -0.209 \\
0.5 & 10.0 & 0.608 & -0.356 & 9.458 & -0.252 & 0.820 & -0.325 & 13.420 & -0.213 \\
\hline 0.4 & 0.5 & 0.168 & -0.403 & 4.423 & -0.264 & 0.484 & -0.292 & 7.402 & -0.213 \\
0.4 & 1.0 & 0.294 & -0.366 & 5.418 & -0.260 & 0.610 & -0.291 & 8.715 & -0.213 \\
0.4 & 5.0 & 0.511 & -0.364 & 7.405 & -0.271 & 0.967 & -0.298 & 12.360 & -0.219 \\
0.4 & 10.0 & 0.705 & -0.353 & 7.699 & -0.289 & 1.099 & -0.311 & 13.720 & -0.227 \\
\hline 0.3 & 0.5 & 0.030 & -0.590 & 1.320 & -0.400 & 0.472 & -0.319 & 7.289 & -0.232 \\
0.3 & 1.0 & 0.057 & -0.552 & 2.226 & -0.364 & 0.615 & -0.315 & 8.615 & -0.232 \\
0.3 & 5.0 & 0.226 & -0.468 & 5.777 & -0.305 & 1.025 & -0.318 & 12.170 & -0.239 \\
0.3 & 10.0 & 0.370 & -0.442 & 6.772 & -0.308 & 1.171 & -0.331 & 13.640 & -0.247 \\
\hline \hline
\end{tabular}

Table 11. Parallel flow fitted parameters of power law functions for square (left) and staggered (right) configurations. 


\section{Acknowledgements}

The authors would like to acknowledge the financial support of the Welsh Government and the European Regional Development Fund (ERDF) provided via the ASTUTE 2020 operation.

\section{NOMENCLATURE}

A Total fibre membrane area.

$A_{\mathrm{f}} \quad$ Frontal fluid area.

$A_{\text {surf }}$ Surface area of fibre resisting the flow.

C Concentration.

$C^{*} \quad$ Normalised concentration.

$C_{\mathrm{f}} \quad$ Concentration along fibre surface.

$C_{\text {in }} \quad$ Concentration at inlet.

$c_{0} \quad$ Rate of rouleaux zero shear.

$c_{\infty} \quad$ Rate of rouleaux at infinite shear.

$\mathrm{CO}_{2}$ Carbon dioxide.

D Rate-of-strain tensor.

$D_{\text {eff }} \quad$ Effective diffusion coefficient.

$D_{\mathrm{rbc}} \quad$ Red blood cell diffusion coefficient.

d Fibre diameter.

$d_{h} \quad$ Hydraulic diameter.

$e \quad$ Relative error.

$G z \quad$ Graetz number.

$h \quad$ Blood oxygenator height.

$H_{\mathrm{b}} \quad$ Haemoglobin.

$H_{\mathrm{ct}} \quad$ Haematocrit.

$k \quad$ Overall mass transfer coefficient.

$\bar{k} \quad$ Surface averaged mass transfer coefficient.

$k_{\mathrm{L}} \quad$ Local mass transfer coefficient.

$K_{\perp}^{*} \quad$ Non-dimensional transverse flow permeability.

$K_{\|}^{*} \quad$ Non-dimensional parallel flow permeability.

$l \quad$ Blood oxygenator length.

$L \quad$ Fibre length.

$L_{\mathrm{f}} \quad$ Length of fibre in blood oxygenator.

$n \quad$ Hill exponent.

n Unit outward normal. 
$N_{\mathrm{f}} \quad$ Maximum number of fibres perpendicular to the flow direction.

$\mathrm{O}_{2} \quad$ Oxygen.

$P \quad$ Pressure.

$Q \quad$ Volumetric flow rate.

Re Reynolds number.

$r \quad$ Fibre radius.

$S \quad$ Saturation.

Sc Schmidt number.

Sh Sherwood number.

$S h_{\mathrm{L}} \quad$ Local Sherwood number.

$\overline{S h} \quad$ Surface averaged Sherwood number.

$\overline{S h}_{\perp} \quad$ Surface averaged Sherwood number for transverse flow.

$\overline{S h}_{\|} \quad$ Surface averaged Sherwood number for parallel.

$S_{\mathrm{p}} \quad$ Fibre spacing.

$\boldsymbol{u} \quad$ Velocity vector.

$\bar{u} \quad$ Averaged velocity.

$u_{x} \quad$ Velocity in $x$-axis.

$u_{y} \quad$ Velocity in $y$-axis.

$u_{z} \quad$ Velocity in $z$-axis.

$\dot{V} \quad$ Gas transfer rate.

$V_{\mathrm{B}} \quad$ Blood volume in packed fibre.

w Blood oxygenator width.

$\mu_{\mathrm{p}} \quad$ Blood plasma viscosity.

$\rho \quad$ Fluid density.

$\alpha \quad$ Gas solubility.

$\boldsymbol{\nabla} \quad$ Gradient operator.

$\beta \quad$ Hill parameter.

$\mathscr{P} \quad$ Partial pressure.

$\varepsilon \quad$ Porosity.

$\dot{\gamma}_{c} \quad$ Characteristic shear rate.

$\dot{\gamma}_{\mathrm{r}} \quad$ Shear rate ratio.

$\dot{\gamma}_{\text {crit }}$ Critical shear rate.

$\dot{\gamma} \quad$ Shear rate.

$\psi \quad$ Variable.

$\tau \quad$ Viscous stress tensor. 


\section{References}

[1] Gaylor, J., 1988. "Membrane oxygenators: current developments in design and application". Journal of Biomedical Engineering, 10(6), pp. $541-547$.

[2] Iwahashi, H., Yuri, K., and Nosé, Y., 2004. "Development of the oxygenator: past, present, and future". Journal of Artificial Organs, 7(3), pp. $111-120$.

[3] Federspiel, W. J., and Henchir, K. A., 2014. Lung, Artificial: Basic Principles And Current Applications, 2nd ed.

[4] Lipnizki, F., and Field, R. W., 2001. "Mass transfer performance for hollow fibre modules with shell-side axial feed flow: using an engineering approach to develop a framework". Journal of Membrane Science, 193(2), pp. 195 - 208.

[5] Wickramasinghe, S. R., Semmens, M. J., and Cussler, E. L., 1991. "Better hollow fiber contactors". Journal of Membrane Science, 62(3), pp. $371-388$.

[6] Wickramasinghe, S. R., Semmens, M. J., Zhang, T., and L., C. E., 1992. "Mass transfer in various hollow fiber geometries". Journal of Membrane Science, 69(3), pp. 235 - 250.

[7] Wickramasinghe, S. R., and Han, B., 2002. "Mass and momentum transfer in commercial blood oxygenators". Desalination, 148(13), pp. $227-233$.

[8] Wickramasinghe, S. R., Garcia, J. D., and Han, B., 1992. "Mass and momentum transfer in hollow fibre blood oxygenators”. Journal of Membrane Science, 69(3), pp. 235 - 250.

[9] Wickramasinghe, S. R., and Han, B., 2005. "Designing microporous hollow fibre blood oxygenators". Chemical Engineering Research and Design, 83(3), pp. $256-267$.

[10] Vaslef, S. N., Cook, K. E., Leonard, R. J., Mockros, L. F., and Anderson, R. W., 1994. "Design and evaluation of a new, low pressure loss, implantable artificial lung”. ASAIO Journal, 40(3), pp. $522-526$.

[11] Vaslef, S. N., Mockros, L. F., Anderson, R. W., and Leonard, R. J., 1994. "Use of a mathematical model to predict oxygen transfer rates in hollow fiber membrane oxygenators". ASAIO Journal, 40(4), pp. 990 - 996.

[12] Nagase, K., Kohori, F., and Sakai, K., 2005. "Oxygen transfer performance of a membrane oxygenator composed of crossed and parallel hollow fibers". Biochemical Engineering Journal, 24(2), pp. 105 - 113.

[13] Dierickx, P. W., De Wachter, D. S., De Somer, F., Van Nooten, G., and Verdonck, P. R., 2001. "Mass transfer characteristics of artificial lungs". The International Journal of Artificial Organs, 47(6), pp. 628 - 633.

[14] Gabelman, A., and Hwang, S.-T., 1999. "Hollow fiber membrane contactors". Journal of Membrane Science, 159(12), pp. $61-106$.

[15] Federspiel, W. J., Hewitt, T. J., and Hattler, B. G., 2000. "Experimental evaluation of a model for oxygen exchange in a pulsating intravascular artificial lung”. Annals of Biomedical Engineering, 28(2), pp. 160 - 167.

[16] Matsuda, N., and Sakai, K., 2000. "Blood flow and oxygen transfer rate of an outside blood flow membrane oxygenator". Journal of Membrane Science, 170(2), pp. $153-158$.

[17] Svitek, R. G., and Federspiel, W. J., 2008. "A mathematical model to predict $\mathrm{CO}_{2}$ removal in hollow fiber membrane oxygenators". Annals of Biomedical Engineering, 36(6), pp. $992-1003$.

[18] Zhang, J., Nolan, T., Zhang, T., Griffith, B. P., and Wu, Z. J. J., 2007. "Characterization of membrane blood oxygenation 
devices using computational fluid dynamics”. Journal of Membrane Science, 288(12), pp. 268 - 279.

[19] Fill, B., Gartner, M., Johnson, G., Horner, M., and Ma, J., 2008. “Computational fluid flow and mass transfer of a functionally integrated pediatric pump-oxygenator configuration”. The International journal of artificial organs, 54(2), pp. $214-219$.

[20] Zhang, J., Taskin, M. E., Koert, A., Zhang, T., Gellman, B., Dasse, K. A., Gilbert, R. J., Griffith, B. P., and Wu, Z. J., 2009. "Computational design and in vitro characterization of an integrated maglev pump-oxygenator". Artificial Organs, 33(10), pp. $805-817$.

[21] Baker, D. A., Holte, J. E., and Patankar, S. V., 1991. "Computationally two-dimensional finite-difference model for hollow-fibre blood-gas exchange devices". Medical and Biological Engineering and Computing, 29(5), pp. 482 - 488.

[22] Dierickx, P. W., De Wachter, D. S., and Verdonck, P. R., 2000. "Blood flow around hollow fibers". The International Journal of Artificial Organs, 23(9), pp. $610-617$.

[23] Dierickx, P. W., De Wachter, D. S., and Verdonck, P. R., 2001. “Two-dimensional finite element model for oxygen transfer in cross-flow hollow fiber membrane artificial lungs". The International Journal of Artificial Organs, 24(9), pp. $628-635$.

[24] Taskin, M. E., Fraser, K. H., Zhang, T., Griffith, B. P., and Wu, Z. J., 2010. "Micro-scale modeling of flow and oxygen transfer in hollow-fiber membrane bundle”. Journal of Membrane Science, 362(1), pp. 172 - 183.

[25] Zierenberg, J. R., Fujioka, H., Suresh, V., Bartlett, R. H., Hirschl, R. B., and Grotberg, J. B., 2006. "Pulsatile flow and mass transport past a circular cylinder”. Physics of Fluids, 18(1), pp. 1 - 15.

[26] Zierenberg, J. R., Fujioka, H., Hirschl, R. B., Bartlett, R. H., and Grotberg, J. B., 2007. "Pulsatile blood flow and oxygen transport past a circular cylinder”. Journal of Biomechanical Engineering, 129(2), pp. 202 - 215.

[27] Chan, K., Fujioka, H., Bartlett, R. H., and Grotberg, J. B., 2006. "Pulsatile flow and mass transport over an array of cylinders: Gas transfer in a cardiac-driven artificial lung”. Journal of Biomechanical Engineering, 128(1), pp. 85 - 96.

[28] Zierenberg, J. R., Fujioka, H., Hirschl, R. B., Bartlett, R. H., and Grotberg, J. B., 2009. “Oxygen and carbon dioxide transport in time-dependent blood flow past fiber rectangular arrays". Physics of Fluids, 21(3), pp. 1 - 19.

[29] Yang, M.-C., and Cussler, E. L., 1986. “Designing hollow-fiber contactors”. AIChE Journal, 32(11), pp. 1910 - 1916.

[30] Vaslef, S. N., Mockros, L. F., Cook, K. E., Leonard, R. J., Sung, J., and Anderson, R. W., 1994. “Computer-assisted design of an implantable, intrathoracic artificial lung”. Artificial Organs, 18(11), pp. 813 - 817.

[31] Low, K. W. Q., van Loon, R., Rolland, S. A., and Sienz, J., 2016. "Pore-scale modelling of non-Newtonian shearthinning fluids in blood oxygenator design”. Journal of Biomechanical Engineering, 138(5), pp. 051001-1 - 05100114.

[32] Marcinkowska-Gapińska, A., Gapinski, J., Elikowski, W., Jaroszyk, F., and Kubisz, L., 2007. "Comparison of three rheological models of shear flow behavior studied on blood samples from post-infarction patients". Medical \& Biological Engineering \& Computing, 45(9), pp. 837 - 844.

[33] Quemada, D., 1978. "Rheology of concentrated disperse systems II. a model for non-Newtonian shear viscosity in steady flows". Rheologica Acta, 17(6), pp. $632-642$. 
[34] Chan, K., Fujioka, H., Bartlett, R. H., and Grotberg, J. B., 2007. "Pulsatile blood flow and gas exchange across a cylindrical fiber array”. Journal of Biomechanical Engineering, 129(5), pp. 676 - 687.

[35] Singh, M. P., Sharan, M., and Aminataei, A., 1989. "Development of mathematical formulae for $\mathrm{O}_{2}$ and $\mathrm{CO}_{2}$ dissociation curves in the blood". Mathematical Medicine and Biology, 6(1), pp. $25-46$.

[36] Dash, R. K., and Bassingthwaighte, J., 2010. "Erratum to: $\mathrm{Blood} \mathrm{HbO}_{2}$ and $\mathrm{HbCO}_{2}$ dissociation curves at varied $\mathrm{O}_{2}$, $\mathrm{CO}_{2}$, pH, 2,3-DPG and temperature levels". Annals of Biomedical Engineering, 38(4), pp. 1683 - 1701.

[37] Incropera, F. P., DeWitt, D. P., Bergman, T. L., and Lavine, A. S., 2007. Fundamentals of Heat and Mass Transfer, 6th ed. John Wiley \& Sons.

[38] Bird, R. B., Stewart, W. E., and Lightfoot, E. N., 2006. Transport Phenomena, 2nd ed. John Wiley \& Sons, Inc.

[39] Woerlee, G., 1988. "Blood gases arterial and venous". In Common Perioperative Problems and the Anaesthetist, Vol. 18 of Developments in Critical Care Medicine and Anesthesiology. Springer Netherlands, pp. 150 - 153.

[40] ANSYS INC., 2011. ANSYS Documentations., release 14.5 ed.

[41] Johnston, B. M., Johnston, P. R., Corney, S., and Kilpatrick, D., 2004. "Non-Newtonian blood flow in human right coronary arteries: steady state simulations". Journal of Biomechanics, 37(5), pp. 709 - 720.

[42] Gebart, B. R., 1992. "Permeability of unidirectional reinforcements for RTM". Journal of Composite Materials, 26(8), pp. $1100-1133$.

[43] Tamayol, A., and Bahrami, M., 2010. "Parallel flow through ordered fibers: an analytical approach". Journal of Fluids Engineering, 132(11), pp. $1-7$.

[44] Coulaud, O., Morel, P., and Caltagirone, J. P., 1988. "Numerical modelling of nonlinear effects in laminar flow through a porous medium”. Journal of Fluid Mechanics, 190, 5, pp. 393 - 407.

[45] Leverett, L., Hellums, J., Alfrey, C., and Lynch, E., 1972. "Red blood cell damage by shear stress". Biophysical Journal, 12(3), pp. $257-273$.

[46] Hellums, J. D., 1994. “1993 Whitaker lecture: Biorheology in thrombosis research”. Annals of Biomedical Engineering, 22(5), pp. $445-455$.

[47] Hochareon, P., Manning, K. B., Fontaine, A. A., Tarbell, J. M., and Deutsch, S. "Correlation of in vivo clot deposition with the flow characteristics in the 50 cc Penn State artificial heart: a preliminary study". ASAIO Journal, 50.

[48] Alemu, Y., and Bluestein, D., 2007. "Flow-induced platelet activation and damage accumulation in a mechanical heart valve: Numerical studies". Artificial Organs, 31(9), pp. 677 - 688.

[49] Fraser, K. H., Zhang, T., Taskin, M. E., Griffith, B. P., and Wu, Z. J., 2012. "A quantitative comparison of mechanical blood damage parameters in rotary ventricular assist devices: Shear stress, exposure time and hemolysis index". Journal of Biomechanical Engineering, 134(8), pp. 081002-1 - 081002-11.

[50] Costello, M., Fane, A., Hogan, P., and Schofield, R., 1993. "The effect of shell side hydrodynamics on the performance of axial flow hollow fibre modules”. Journal of Membrane Science, 80(1), pp. 1 - 11.

[51] Fasano, A., Santos, R., and Sequeira, A., 2012. "Blood coagulation: A puzzle for biologists, a maze for mathematicians”. In Modeling of Physiological Flows, D. Ambrosi, A. Quarteroni, and G. Rozza, eds., Vol. 5 of MS\&A Modeling, 
Simulation and Applications. Springer Milan, pp. 41 - 75 . 